\title{
A!
}

This is an electronic reprint of the original article.

This reprint may differ from the original in pagination and typographic detail.

Partanen, Mikko; Tulkki, Jukka

\section{Mass-polariton theory of light in dispersive media}

Published in:

Physical Review A

DOI:

10.1103/PhysRevA.96.063834

Published: 26/12/2017

Document Version

Publisher's PDF, also known as Version of record

Please cite the original version:

Partanen, M., \& Tulkki, J. (2017). Mass-polariton theory of light in dispersive media. Physical Review A, 96(6), 114. [063834]. https://doi.org/10.1103/PhysRevA.96.063834

This material is protected by copyright and other intellectual property rights, and duplication or sale of all or part of any of the repository collections is not permitted, except that material may be duplicated by you for your research use or educational purposes in electronic or print form. You must obtain permission for any other use. Electronic or print copies may not be offered, whether for sale or otherwise to anyone who is not an authorised user. 


\title{
Mass-polariton theory of light in dispersive media
}

\author{
Mikko Partanen and Jukka Tulkki \\ Engineered Nanosystems Group, School of Science, Aalto University, P.O. Box 11000, 00076 Aalto, Finland
}

(Received 16 October 2017; published 26 December 2017)

\begin{abstract}
We have recently shown that the electromagnetic pulse in a medium is made of mass-polariton (MP) quasiparticles, which are quantized coupled states of the field and an atomic mass density wave (MDW) [M. Partanen et al., Phys. Rev. A 95, 063850 (2017)]. In this work, we generalize the MP theory of light for dispersive media assuming that absorption and scattering losses are very small. Following our previous work, we present two different approaches to the coupled state of light: (1) the MP quasiparticle theory, which is derived by only using the fundamental conservation laws and the Lorentz transformation; (2) the classical optoelastic continuum dynamics (OCD), which is a generalization of the electrodynamics of continuous media to include the dynamics of the medium under the influence of optical forces. We show that the total momentum and the transferred mass of the light pulse can be determined in a straightforward way if we know the field energy of the pulse and the dispersion relation of the medium. In analogy to the nondispersive case, we also find unambiguous correspondence between the MP and OCD theories. For the coupled MP state of a single photon and the medium, we obtain the total MP momentum $p_{\mathrm{MP}}=n_{\mathrm{p}} \hbar \omega / c$, where $n_{\mathrm{p}}$ is the phase refractive index. The field's share of the MP momentum is equal to $p_{\text {field }}=\hbar \omega /\left(n_{\mathrm{g}} c\right)$, where $n_{\mathrm{g}}$ is the group refractive index and the share of the MDW is equal to $p_{\mathrm{MDW}}=p_{\mathrm{MP}}-p_{\text {field }}$. Thus, as in a nondispersive medium, the total momentum of the MP is equal to the Minkowski momentum and the field's share of the momentum is equal to the Abraham momentum. We also show that the correspondence between the MP and OCD models and the conservation of momentum at interfaces gives an unambiguous formula for the optical force. The dynamics of the light pulse and the related MDW lead to nonequilibrium of the medium and to relaxation of the atomic density by sound waves in the same way as for nondispersive media. We also carry out simulations for optimal measurements of atomic displacements related to the MDW in silicon. In the simulations, we consider different waveguide cross sections and optical pulse widths and account for the breakdown threshold irradiance of materials. We also compare the MP theory to previous theories of the momentum of light in a dispersive medium. We show that our generalized MP theory resolves all the problems related to the Abraham-Minkowski dilemma in a dispersive medium.
\end{abstract}

DOI: 10.1103/PhysRevA.96.063834

\section{INTRODUCTION}

Previous theories of light in a medium have neglected the possibility of an associated mass density wave (MDW) formed by small atomic movements caused by the optical force that is alternately accelerating and decelerating medium atoms. We have recently shown that the MDW is an unavoidable part of the consistent theory of light in a medium [1]. In the single-photon picture, the coupling of the electromagnetic field to the atomic MDW gives rise to mass-polariton (MP) quasiparticles, which are covariant coupled states of the field and matter having a nonzero rest mass [1]. The coupled state of the field and matter can also be described by using classical optoelastic continuum dynamics (OCD) [1]. In the OCD model, the electrodynamics of continuous media [2] is generalized to include the coupling between the field and matter and the related continuum dynamics of the medium.

Accounting for the MDW coupled to the electromagnetic field, the photon mass drag effect has been shown [1] to resolve the centennial Abraham-Minkowski controversy of optical momentum in a medium [3-14]. This controversy has its origin in the formulation of two rivaling momentum densities for light by Abraham, $\mathbf{g}_{\mathrm{A}}=\mathbf{E} \times \mathbf{H} / c^{2}[15,16]$, and by Minkowski, $\mathbf{g}_{\mathbf{M}}=\mathbf{D} \times \mathbf{B}$ [17], where $c$ is the speed of light in vacuum, $\mathbf{E}$ and $\mathbf{H}$ are the electric- and magnetic-field strengths, and $\mathbf{D}$ and $\mathbf{B}$ are the electric and magnetic flux densities. For a nondispersive medium, the momentum densities $\mathbf{g}_{\mathrm{A}}$ and $\mathbf{g}_{\mathrm{M}}$ correspond to the single-photon momenta $p_{\mathrm{A}}=\hbar \omega /(n c)$ or $p_{\mathrm{M}}=n \hbar \omega / c$, respectively, where $\hbar$ is the reduced Planck constant, $\omega$ is the angular frequency of the field, and $n$ is the refractive index of the medium. In order to determine the momentum of light in a medium, several experimental setups have been introduced [18-28] but with partly controversial results. In the recently developed MP theory [1], the Abraham momentum $p_{\mathrm{A}}$ is the momentum of the electromagnetic field of the coupled MP state while the difference $p_{\mathrm{M}}-p_{\mathrm{A}}$ is carried by the MDW. The total MP momentum is then of the Minkowski form $p_{\mathrm{M}}=n \hbar \omega / c$.

The initial derivation of the MP theory in Ref. [1] assumed a nondispersive medium. In this work, we generalize the MP quasiparticle model based on the conservation laws and the Lorentz transformation for dispersive media. Following Ref. [1], we also present the complementary classical OCD model, which we have generalized for a dispersive medium. The OCD model uses the optoelastic force density to calculate the coupled Newtonian dynamics of the field and the medium [1]. The calculations show that the quasiparticle and continuum dynamics models are in full agreement in the limit of a monochromatic field, i.e., when the photon picture becomes reasonable, and also in the limit of weak dispersion.

This paper is organized as follows: Section II presents a brief summary of the most conclusive experiments to measure the momentum of light in dispersive media. Section III reviews the well-known principles of the dispersion relations and the solution of the electric and magnetic fields of a light pulse. This is followed by presenting the OCD model generalized for dispersive media in Sec. IV and the related complementary 
MP quasiparticle model in Sec. V. In this work, we consider the OCD model first since it is for most readers easier to approach being based on the familiar concepts of Maxwell's and Newton's theories. However, the theories are independent and the reader can also start from the MP quasiparticle model. Section VI presents the OCD simulations of a Gaussian light pulse propagating in linearly and nonlinearly dispersive media. To facilitate the planning of possible experiments of the transferred mass of the MDW, we also compute the atomic displacements due to the MDW in a schematic silicon waveguide structure. The results of the OCD and MP quasiparticle models are compared in Sec. VII. We also compare our theory to selected previous experiments and theories that have been used to determine the momentum of light in a dispersive medium. Finally, conclusions are drawn in Sec. VIII.

\section{BRIEF SUMMARY OF EXPERIMENTS}

The most conclusive set of experiments to measure the momentum of light in a medium were started in 1954 by Jones and Richards [20] who studied the pressure exerted by light on a reflector immersed in a liquid with known refractive index. By performing the experiment with a number of liquids of varying refractive index, they showed with $1.2 \%$ precision that the pressure on a reflector immersed in a liquid scales linearly with the refractive index. The experiment was repeated in 1978 by Jones and Leslie [21] with $0.05 \%$ precision. A more than tenfold improvement in precision was possible by using a laser as a light source and multilayer reflectors of high reflectivity and low absorption. The accuracy obtained was sufficient to conclusively show that the force on the mirror is directly proportional to the phase refractive index $n_{\mathrm{p}}$ and not to the group refractive index $n_{\mathrm{g}}$. A principally identical schematic experimental setup is illustrated in Fig. 1. If the force $\mathbf{F}_{2}$ on the perfect lossless reflector results from the single-photon impulses $\Delta \mathbf{p}_{i}$ in time $\Delta t$, we obtain $\mathbf{F}_{2}=$ $\sum_{i} \Delta \mathbf{p}_{i} / \Delta t$. Then the experiment unambiguously supports the Minkowski formula $p=n_{\mathrm{p}} \hbar \omega / c$ [5-7], provided that we

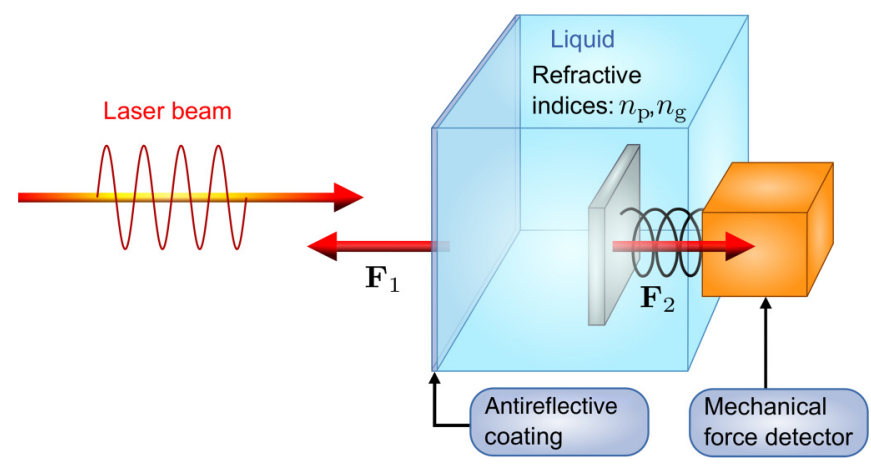

FIG. 1. Schematic illustration of an experimental setup for the measurement of the electromagnetic forces due to a light beam in a dispersive medium. Light enters from vacuum to a liquid container with antireflective coating. Inside the liquid having phase and group refractive indices $n_{\mathrm{p}}$ and $n_{\mathrm{g}}$, light is fully reflected from a mirror attached to a detector that measures the resulting force $\mathbf{F}_{2} . \mathbf{F}_{1}$ is a recoil force that balances the conservation law of momentum at the interface. know the intensity and the frequency of a monochromatic laser beam. There exist also other experiments that have been interpreted to support either the Minkowski or Abraham momentum [18,19,22-27]. In these experiments, the relation of the measured force or other quantity to the momentum of light is much more subtle and analyzing these experiments using our theory is a topic of a separate work.

If we consider light in a dispersive medium as a coupled state of the field and matter, we are expected to be able to apply the de Broglie wavelength in the analysis of diffraction experiments. In the diffraction experiments, one obtains the de Broglie wavelength which is related to the momentum of the coupled state as $\lambda=h / p$, where $h$ is the (nonreduced) Planck constant. Since numerous diffraction experiments have confirmed that the wavelength fulfilling the diffraction condition is given by $\lambda=\lambda_{0} / n_{\mathrm{p}}$, where $\lambda_{0}$ is the vacuum wavelength, we obtain $p=n_{\mathrm{p}} \hbar \omega_{0} / c$, which is again of the Minkowski form. Note that so far there are no reported measurements of the transferred mass of a light pulse.

\section{SOLUTION OF FIELDS IN DISPERSIVE MEDIA}

\section{A. General dispersion}

In dispersive media, the phase velocity and the phase refractive index depend on frequency $\omega(k)=c k / n_{\mathrm{p}}(\omega)$, where $n_{\mathrm{p}}(\omega)$ is the frequency-dependent phase refractive index. The phase velocity is given by $v_{\mathrm{p}}(\omega)=c / n_{\mathrm{p}}(\omega)=\omega(k) / k$ and the group velocity by the formula $v_{\mathrm{g}}(\omega)=c / n_{\mathrm{g}}(\omega)=\partial \omega(k) / \partial k$, where $n_{\mathrm{g}}(\omega)$ is the group refractive index.

The most general forms of the electric and magnetic fields of a linearly polarized one-dimensional light pulse propagating in $x$ direction in a dispersive medium can be written as [29]

$$
\begin{aligned}
& \mathbf{E}(\mathbf{r}, t)=\operatorname{Re}\left[\int_{-\infty}^{\infty} \tilde{E}(k) e^{i[k x-\omega(k) t]} d k\right] \hat{\mathbf{y}}, \\
& \mathbf{H}(\mathbf{r}, t)=\operatorname{Re}\left[\int_{-\infty}^{\infty} \tilde{H}(k) e^{i[k x-\omega(k) t]} d k\right] \hat{\mathbf{z}},
\end{aligned}
$$

where $\hat{\mathbf{y}}$ and $\hat{\mathbf{z}}$ are unit vectors with respect to $y$ and $z$ axes and $\tilde{E}(k)$ and $\tilde{H}(k)$ are the Fourier components of the electric and magnetic fields. The field components are related to each other by $\tilde{H}(k)=\sqrt{\varepsilon[\omega(k)] / \mu[\omega(k)]} \tilde{E}(k)$, where $\varepsilon(\omega)$ and $\mu(\omega)$ are the frequency-dependent permittivity and permeability of the medium. These are related to the phase refractive index as $\varepsilon(\omega) \mu(\omega)=\varepsilon_{0} \mu_{0} n_{\mathrm{p}}(\omega)^{2}$, where $\varepsilon_{0}$ and $\mu_{0}$ are the permittivity and permeability of the vacuum respectively. The electric and magnetic fields in Eqs. (1) and (2) are exact solutions of Maxwell's equations.

\section{B. Linear dispersion}

We first investigate a light pulse in a dispersive medium, where the dispersion relation is effectively linear near the central frequency $\omega_{0}$ containing the first terms of the Taylor expansion of $\omega(k)$ as

$$
\omega(k) \approx \omega_{0}+\left(c / n_{\mathrm{g}}\right)\left(k-k_{0, \mathrm{med}}\right),
$$

where $k_{0 \text {,med }}=n_{\mathrm{p}} k_{0}$ is the wave number corresponding to $\omega_{0}$ in the medium, $k_{0}=\omega_{0} / c$ is the wave number in vacuum, $n_{\mathrm{p}}=n_{\mathrm{p}}\left(\omega_{0}\right)$ is the phase refractive index for $\omega_{0}$, and the group 
refractive index $n_{\mathrm{g}}$ is constant. The linear dispersion relation in Eq. (3) is a good approximation for any general dispersion relation if the frequency spread of the wave packet is relatively small, the dispersion relation does not have sharp variations due to resonances, and if the wave packet does not travel over very long distances. Otherwise, higher-order terms in the Taylor expansion of $\omega(k)$ also become important.

For frequencies deviating from $\omega_{0}$, the linear dispersion relation in Eq. (3) defines the frequency-dependent phase refractive index as

$$
n_{\mathrm{p}}(\omega)=n_{\mathrm{g}}+\left(n_{\mathrm{p}}-n_{\mathrm{g}}\right) \frac{\omega_{0}}{\omega} .
$$

The linear form of the dispersion relation in Eq. (3) is known to be the most general form of the dispersion relation, which does not lead to the distortion of the pulse envelope while the pulse propagates.

We assume a Gaussian light pulse with $\tilde{E}(k)=$ $\tilde{E}_{0} e^{-\left[\left(k-n_{\mathrm{p}} k_{0}\right) /\left(n_{\mathrm{p}} \Delta k_{0}\right)\right]^{2} / 2}$ where $\tilde{E}_{0}$ is a normalization factor and $\Delta k_{0}$ is the standard deviation of the wave number in vacuum related to the pulse width in the $x$ direction as $\Delta x=1 /\left(\sqrt{2} n_{\mathrm{p}} \Delta k_{0}\right)$. The corresponding standard deviation in time is then $\Delta t=n_{\mathrm{p}} \Delta x / c=1 /\left(\sqrt{2} \Delta k_{0} c\right)$ and the full width at half maximum is $\Delta t_{\mathrm{FWHM}}=2 \sqrt{2 \ln 2} \Delta t$. Using Eq. (1) and the linear dispersion relation in Eq. (3), the electric field then becomes

$$
\begin{aligned}
\mathbf{E}(\mathbf{r}, t)= & \sqrt{2 \pi} n_{\mathrm{p}} \Delta k_{0} \tilde{E}_{0} \cos \left[n_{\mathrm{p}} k_{0}\left(x-\frac{c t}{n_{\mathrm{p}}}\right)\right] \\
& \times e^{-\left(n_{\mathrm{p}} \Delta k_{0}\right)^{2}\left(x-c t / n_{\mathrm{g}}\right)^{2} / 2} \hat{\mathbf{y}} .
\end{aligned}
$$

The normalization factor $\tilde{E}_{0}$ in Eq. (5) can be determined so that the integral of the corresponding instantaneous energy density over $x$ gives $U_{0} / A$, where $A$ is a cross-sectional area and $U_{0}$ is the total energy of the light pulse.

An example of the electric field of a Gaussian light pulse given in Eq. (5) is presented in Fig. 2. The envelope function described by the exponential factor in Eq. (5) propagates at the group velocity $v_{\mathrm{g}}=c / n_{\mathrm{g}}$ while the individual peaks and troughs inside the wave envelope propagate at the phase velocity $v_{\mathrm{p}}=c / n_{\mathrm{p}}$. In other words, the phase velocity $v_{\mathrm{p}}(\omega)$ describes the propagation velocity of individual frequency components while the amplitudes of the frequency components add up to produce a wave packet, which propagates at the group velocity [29]. Therefore, the total energy of the wave packet propagates at the group velocity.

\section{Nonlinear dispersion}

In general, the linear dispersion relation above cannot provide a complete description of dispersion close to resonances or in the case of large frequency ranges. Therefore, following some previous works on the Abraham-Minkowski controversy [30], we study as an example of nonlinear dispersion a simple Lorentz model for a dielectric medium with a single resonance frequency $\omega_{\mathrm{r}}$ and zero damping factor [31]. The imaginary part of the refractive index can be assumed zero at $\omega_{0}$ and the real

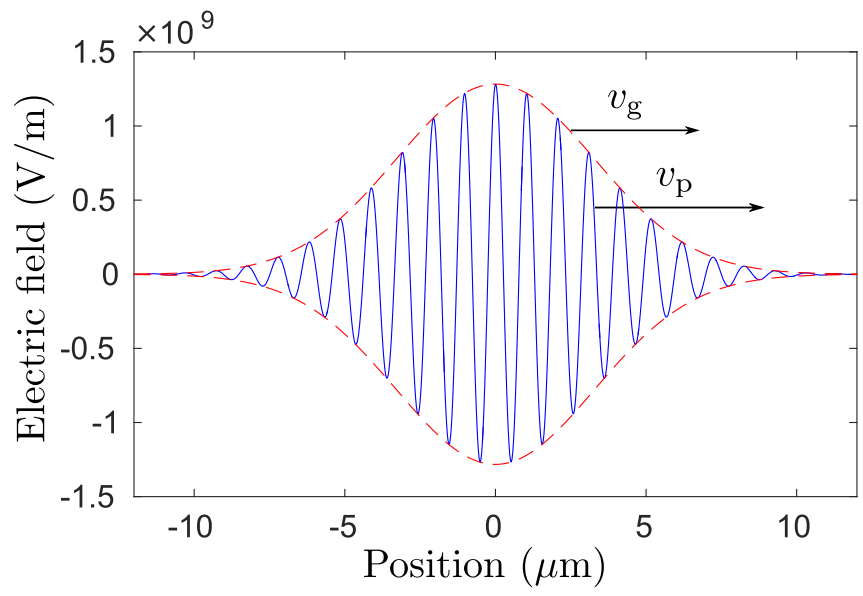

FIG. 2. Example of the electric field and its envelope function in the case of an ultrashort Gaussian light pulse of vacuum wavelength $\lambda_{0}=1550 \mathrm{~nm}, \Delta t_{\mathrm{FWHM}}=27 \mathrm{fs}$, and energy $U_{0}=1 \mu \mathrm{J}$ per crosssectional area of diameter $d=100 \mu \mathrm{m}$. The phase and group refractive indices for the central frequency in a linearly dispersive medium are assumed to be $n_{\mathrm{p}}=1.5$ and $n_{\mathrm{g}}=2$. The wave envelope propagates at the group velocity $v_{\mathrm{g}}=c / n_{\mathrm{g}}$ while the individual peaks and troughs inside the wave envelope propagate at the phase velocity $v_{\mathrm{p}}=c / n_{\mathrm{p}}$.

part of the refractive index is given by [30]

$$
n_{\mathrm{p}}(\omega)=\sqrt{1+\frac{\omega_{\mathrm{p}}^{2}}{\omega_{\mathrm{r}}^{2}-\omega^{2}}}
$$

where $\omega_{\mathrm{p}}$ is a model parameter. The dispersion equation $k=$ $n_{\mathrm{p}}(\omega) \omega / c$ then takes the quadratic form [30]

$$
\omega^{4}-\left(\omega_{\mathrm{p}}^{2}+\omega_{\mathrm{r}}^{2}+k^{2} c^{2}\right) \omega^{2}+k^{2} c^{2} \omega_{\mathrm{r}}^{2}=0 .
$$

For each $k$, there are two positive solutions. These solutions are given by [30]

$\omega_{ \pm}=\sqrt{\frac{\omega_{\mathrm{r}}^{2}+\omega_{\mathrm{p}}^{2}+k^{2} c^{2} \pm \sqrt{\left(\omega_{\mathrm{r}}^{2}+\omega_{\mathrm{p}}^{2}+k^{2} c^{2}\right)^{2}-4 k^{2} c^{2} \omega_{\mathrm{r}}^{2}}}{2}}$.

These are called the upper (+) and lower (-) polariton branches and they have been illustrated in Fig. 3. For the wave number $k=n_{\mathrm{p}}\left(\omega_{0}\right) \omega_{0} / c$ corresponding to $\omega_{0}$ with $\omega_{0}>\omega_{\mathrm{r}}$, we obtain $\omega_{+}=\omega_{0}$ and $\omega_{-}=n_{\mathrm{p}}\left(\omega_{0}\right) \omega_{\mathrm{r}}$ and with $\omega_{0}<\omega_{\mathrm{r}}$ we obtain $\omega_{+}=n_{\mathrm{p}}\left(\omega_{0}\right) \omega_{\mathrm{r}}$ and $\omega_{-}=\omega_{0}$. We must restrict to the solution for which $\omega_{i}=\omega_{0}$. The other solution of the dispersion equation has the same wave number, but the frequency is very different from $\omega_{0}$.

The main difference between linear and nonlinear dispersion is that, in the nonlinear case, the envelope of a wave packet becomes distorted as it travels. This follows from the fact that the group velocity $v_{\mathrm{g}}=\partial \omega(k) / \partial k$ is not constant but different for different values of $k$. Therefore, the wavenumber components move at different velocities distorting the envelope of the wave packet. However, if the wave packet has a range of frequencies that is narrow enough compared to the nonlinearity, then $\omega(k)$ is necessarily approximately linear over that narrow range and the pulse distortion is small. 


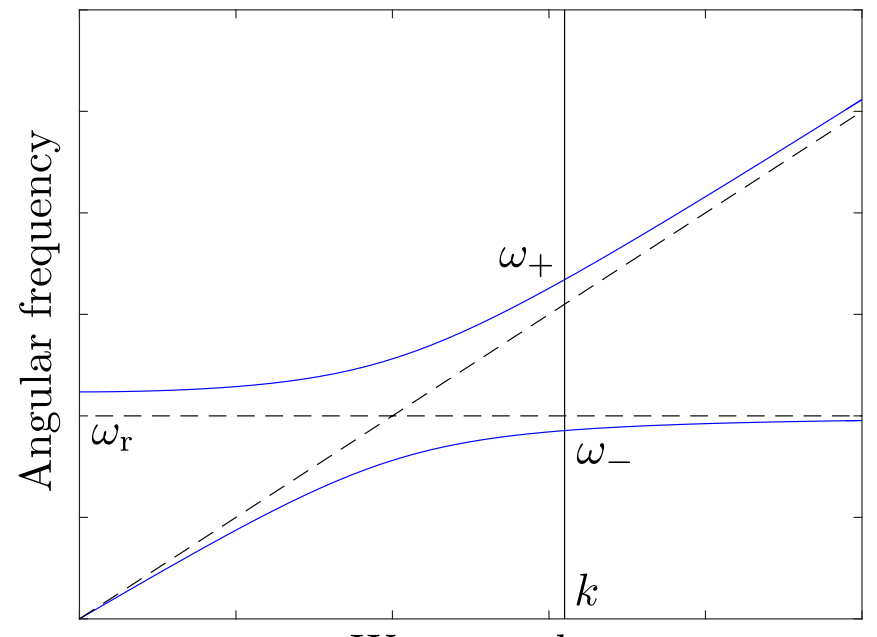

Wave number

FIG. 3. An example of a two-branch polariton dispersion curve with a single resonance frequency $\omega_{\mathrm{r}}$. For the lower branch $\omega<\omega_{\mathrm{r}}$ and for the upper branch $\omega>\omega_{\mathrm{r}}$.

Therefore, in the limit of narrow frequency range, using the linear dispersion relation in Eq. (3) becomes an accurate approximation.

\section{OPTOELASTIC CONTINUUM DYNAMICS}

\section{A. Optical force density}

In previous literature, there has been extensive discussion on the appropriate form of the force density acting on the medium under the influence of time-dependent electromagnetic field [30]. We have recently shown [1] that there is only one form of optical force density that is fully consistent with the MP quasiparticle model and the underlying principles of the special theory of relativity in the case of nondispersive media [1]. We generalize this optical force density for dispersive media by writing

$$
\mathbf{f}_{\mathrm{opt}}(\mathbf{r}, t)=-\varepsilon_{0} n_{\mathrm{g}} \mathbf{E}^{2} \nabla n_{\mathrm{p}}+\frac{n_{\mathrm{p}} n_{\mathrm{g}}-1}{c^{2}} \frac{\partial}{\partial t} \mathbf{E} \times \mathbf{H},
$$

where $\mathbf{E} \times \mathbf{H}=\mathbf{S}$ is the instantaneous Poynting vector. The expression of the optical force density given in Eq. (9) can be at this stage taken as a postulate. We will later on justify it by showing that it is the only form of the optical force density that enables covariant description of the light pulse, fulfills the conservation law of momentum, and is also consistent with the MP quasiparticle model. We have not found this specific form of the optical force density in previous works.

In calculating the optoelastic force field, we assume that the damping of the electromagnetic field due to the transfer of field energy to the kinetic and elastic energies of the medium by the optical force is negligible. Adopting this perturbative approach is justified as the effect of the fields on the dynamical state of the material is such that the back action of the dynamics of the medium on the state of the fields is extremely small. The accuracy of this approximation is estimated in Ref. [1] and the conclusions are valid also for dispersive media if there is no direct optical absorption related, e.g., to the electronic excitation of the medium.

\section{B. Newton's equation of motion}

In the OCD model, the coupling between the field and matter is described by Newton's equation of motion. As the atomic velocities are nonrelativistic, Newton's equation of motion for the mass density of the medium $\rho_{\mathrm{a}}(\mathbf{r}, t)$ is given by

$$
\rho_{\mathrm{a}}(\mathbf{r}, t) \frac{d^{2} \mathbf{r}_{\mathrm{a}}(\mathbf{r}, t)}{d t^{2}}=\mathbf{f}_{\mathrm{opt}}(\mathbf{r}, t)+\mathbf{f}_{\mathrm{el}}(\mathbf{r}, t),
$$

where $\mathbf{r}_{\mathrm{a}}(\mathbf{r}, t)$ is the position- and time-dependent atomic displacement field of the medium, $\mathbf{f}_{\mathrm{opt}}(\mathbf{r}, t)$ is the optical force density experienced by atoms, given in Eq. (9), and $\mathbf{f}_{\mathrm{el}}(\mathbf{r}, t)$ is the elastic force density between atoms that are displaced from their initial equilibrium positions by the optical force density.

Close to equilibrium, the elastic forces between atoms are known to be well described by Hooke's law. In the simple case of a homogeneous isotropic elastic medium, the elastic force density in terms of the material displacement field $\mathbf{r}_{\mathrm{a}}(\mathbf{r}, t)$ is well known to be given by [32]

$$
\mathbf{f}_{\mathrm{el}}(\mathbf{r}, t)=\left(B+\frac{4}{3} G\right) \nabla\left[\nabla \cdot \mathbf{r}_{\mathrm{a}}(\mathbf{r}, t)\right]-G \nabla \times\left[\nabla \times \mathbf{r}_{\mathrm{a}}(\mathbf{r}, t)\right],
$$

where $B$ is the bulk modulus and $G$ is the shear modulus of the medium [33]. In more general anisotropic cubic crystals, Eq. (11) must be replaced with a more general form given by the following set of componentwise equations [34]:

$$
\begin{aligned}
f_{\mathrm{el}, x}= & C_{11} \frac{\partial^{2} r_{\mathrm{a}, x}}{\partial x^{2}}+C_{44}\left(\frac{\partial^{2} r_{\mathrm{a}, x}}{\partial y^{2}}+\frac{\partial^{2} r_{\mathrm{a}, x}}{\partial z^{2}}\right) \\
& +\left(C_{12}+C_{44}\right)\left(\frac{\partial^{2} r_{\mathrm{a}, y}}{\partial x \partial y}+\frac{\partial^{2} r_{\mathrm{a}, z}}{\partial x \partial z}\right), \\
f_{\mathrm{el}, y}= & C_{11} \frac{\partial^{2} r_{\mathrm{a}, y}}{\partial y^{2}}+C_{44}\left(\frac{\partial^{2} r_{\mathrm{a}, y}}{\partial x^{2}}+\frac{\partial^{2} r_{\mathrm{a}, y}}{\partial z^{2}}\right) \\
& +\left(C_{12}+C_{44}\right)\left(\frac{\partial^{2} r_{\mathrm{a}, x}}{\partial x \partial y}+\frac{\partial^{2} r_{\mathrm{a}, z}}{\partial y \partial z}\right), \\
f_{\mathrm{el}, z}= & C_{11} \frac{\partial^{2} r_{\mathrm{a}, z}}{\partial z^{2}}+C_{44}\left(\frac{\partial^{2} r_{\mathrm{a}, z}}{\partial x^{2}}+\frac{\partial^{2} r_{\mathrm{a}, z}}{\partial y^{2}}\right) \\
& +\left(C_{12}+C_{44}\right)\left(\frac{\partial^{2} r_{\mathrm{a}, x}}{\partial x \partial z}+\frac{\partial^{2} r_{\mathrm{a}, y}}{\partial y \partial z}\right),
\end{aligned}
$$

where $C_{11}, C_{12}$, and $C_{44}$ are elastic constants. The forces given in Eqs. (12)-(14) simplify to the case of an isotropic medium in Eq. (11) by substitutions $C_{11}=B+\frac{4}{3} G, C_{12}=B-\frac{2}{3} G$, and $C_{44}=G$.

\section{Energy and momentum of the MP}

For a monochromatic field with angular frequency $\omega_{0}$ in a lossless dispersive medium, the energy and momentum are given by [2]

$$
\begin{gathered}
E_{\text {field }}=\int \frac{1}{2}\left[\frac{d\left(\varepsilon \omega_{0}\right)}{d \omega_{0}} \mathbf{E}^{2}+\frac{d\left(\mu \omega_{0}\right)}{d \omega_{0}} \mathbf{H}^{2}\right] d^{3} r, \\
\mathbf{p}_{\text {field }}=\int \frac{1}{c^{2}} \mathbf{E} \times \mathbf{H} d^{3} r .
\end{gathered}
$$


The momentum density of the field in Eq. (16) is essentially of the Abraham form. The momentum density in Eq. (16) is also justified by the MP quasiparticle model as described below.

The energy density in the integrand of Eq. (15) is known to be accurate only in the limit of a monochromatic field. Here, we use it as an approximation for light pulses. A more accurate but also more complicated expression for the energy density of a finite light pulse in a dispersive medium is given in Ref. [35].

In the same way as done for a nondispersive medium in Ref. [1], it can be easily shown that, in the OCD model, the energy and momentum of the MDW atoms are given by

$$
\begin{gathered}
E_{\mathrm{MDW}}=\int \rho_{\mathrm{MDW}} c^{2} d^{3} r \approx\left(n_{\mathrm{p}} n_{\mathrm{g}}-1\right) E_{\text {field }} \\
\mathbf{p}_{\mathrm{MDW}}=\int \rho_{\mathrm{a}} \mathbf{v}_{\mathrm{a}} d^{3} r=\int \rho_{\mathrm{MDW}} \mathbf{v}_{\mathrm{g}} d^{3} r \approx\left(n_{\mathrm{p}} n_{\mathrm{g}}-1\right) \mathbf{p}_{\text {field }} .
\end{gathered}
$$

Here $\mathbf{v}_{\mathrm{a}}=d \mathbf{r}_{\mathrm{a}} / d t$ is the velocity of atoms, $\mathbf{v}_{\mathrm{g}}$ is the group velocity vector, and the MDW mass density $\rho_{\mathrm{MDW}}$ is given by $\rho_{\text {MDW }}=\rho_{\mathrm{a}}-\rho_{0}$, in which $\rho_{0}$ is the equilibrium mass density of the medium. Thus, the MDW mass density corresponds to the excess mass density in the medium. The total energy and momentum of the MP are given as sums $E_{\mathrm{MP}}=E_{\mathrm{MDW}}+E_{\mathrm{field}}$ and $\mathbf{p}_{\mathrm{MP}}=\mathbf{p}_{\mathrm{MDW}}+\mathbf{p}_{\text {field }}$ resulting in

$$
\begin{gathered}
E_{\mathrm{MP}}=\int\left\{\rho_{\mathrm{MDW}} c^{2}+\frac{1}{2}\left[\frac{d\left(\varepsilon \omega_{0}\right)}{d \omega_{0}} \mathbf{E}^{2}+\frac{d\left(\mu \omega_{0}\right)}{d \omega_{0}} \mathbf{H}^{2}\right]\right\} d^{3} r \\
\mathbf{p}_{\mathrm{MP}}=\int\left(\rho_{\mathrm{a}} \mathbf{v}_{\mathrm{a}}+\frac{1}{c^{2}} \mathbf{E} \times \mathbf{H}\right) d^{3} r
\end{gathered}
$$

Following Appendix B of Ref. [1], it is also straightforward to present the energy and momentum densities in the integrands of Eqs. (15)-(20) using the energy-momentum tensor formalism. The total energy-momentum tensor of the MP can also be correspondingly split into parts related to the electromagnetic field and the MDW.

\section{MASS-POLARITON QUASIPARTICLE MODEL}

In the following, we generalize the MP quasiparticle model of Ref. [1] for dispersive media. To emphasize the role of the MP as an intrinsic covariant state of a single photon coupled to the medium, we neglect for the moment the possible interface effects that occur when the photon enters the medium and instead assume that a photon having a field energy $\hbar \omega_{0}$ is propagating inside the medium. Generalization of the present work for full quantum optical description of the MP is left for future works.

Instead, we use an analogy of a single MP state to a very narrow wave packet in phase space having a central frequency $\omega_{0}$ and field energy $\hbar \omega_{0}$. In the OCD theory, such a wave packet can be made arbitrarily close to a monochromatic wave. Monochromatic components of such a wave packet propagate at the phase velocity $v_{\mathrm{p}}$. First, we assume that the field energy of the wave packet will vanish in the frame propagating with velocity $v_{\mathrm{p}}$ ( $F$ frame). Second, we assume that the frame moving with the group velocity $v_{\mathrm{g}}(R$ frame) is the rest frame of the MP and accordingly the total momentum of the MP becomes zero in this frame. Third, in analogy with the case of a nondispersive medium, we know that the kinetic energy of the atomic MDW is extremely small in the laboratory frame ( $L$ frame), which is the initial rest frame of the medium. As described in Ref. [1], the mass $\delta m$ of the MDW is carried by atoms. Since the total mass of atoms in the MDW is vastly larger than the mass $\delta m$ carried by the MDW, the speed of atoms is very small and, in particular, their kinetic energy is extremely small in comparison with $\hbar \omega_{0}$. Next we determine the total energy and momentum of the MP by requiring that their values in the $L$ frame, $F$ frame, and $R$ frame are related by the Lorentz transformation.

$L$ frame. The total energy of the MP in $L$ frame is given by $E_{\mathrm{MP}}=\hbar \omega_{0}+\delta m c^{2}$. The first term is the assumed fixed field energy. The second term $\delta m$ is the mass energy carried by the MDW. Note that, as discussed above, the kinetic energy of the MDW is negligible in the $L$ frame. The problem to be solved is to determine $\delta m$ and the total momentum $p_{\mathrm{MP}}$ of the MP.

Lorentz transformation. When the $L$ frame energy and momentum of the MP are transformed to any frame moving with constant velocity $v$ with respect to the $L$ frame, their values in the moving frame are given by the Lorentz transformation as

$$
E_{\mathrm{MP}}^{\prime}=\gamma_{v}\left(E_{\mathrm{MP}}-v p_{\mathrm{MP}}\right)=\gamma_{v}\left(\hbar \omega_{0}-v p_{\mathrm{MP}}\right)+\gamma_{v} \delta m c^{2} \text {, }
$$

$$
p_{\mathrm{MP}}^{\prime}=\gamma_{v}\left(p_{\mathrm{MP}}-\frac{v E_{\mathrm{MP}}}{c^{2}}\right)=\gamma_{v}\left(p_{\mathrm{MP}}-\frac{v \hbar \omega_{0}}{c^{2}}-v \delta m\right),
$$

where $\gamma_{v}=1 / \sqrt{1-v^{2} / c^{2}}$ is the Lorentz factor. In the following, we will show that $p_{\mathrm{MP}}$ and $\delta m$ can be determined by investigating Eqs. (21) and (22) in two special inertial frames: the $F$ frame and the $R$ frame.

$F$ frame. First, we observe that, in Eq. (21), the last term on the right represents the transformed mass energy of the MP, while the first term $\hbar \omega_{0}^{\prime}=\gamma_{v}\left(\hbar \omega_{0}-v p_{\mathrm{MP}}\right)$ has its origin entirely in the field energy. In the special case of the $F$ frame, which propagates with the phase velocity $v=v_{\mathrm{p}}=c / n_{\mathrm{p}}$, the frequency and the related field energy become zero as $\hbar \omega_{0}^{\prime} \rightarrow$ 0 . Therefore, we obtain

$$
p_{\mathrm{MP}}=\frac{n_{\mathrm{p}} \hbar \omega_{0}}{c}
$$

which is of the Minkowski form as commonly defined in literature for a dispersive medium [6,7]. Note that in the literature there exists also another rather commonly defined form of the Minkowski momentum given by $p_{\mathrm{M}}=n_{\mathrm{p}}^{2} \hbar \omega_{0} /\left(n_{\mathrm{g}} c\right)$ [36]. These momenta are discussed in more detail in Sec. VII B.

$R$ frame. Second, we consider the special case of the $R$ frame in which the total momentum of the MP is zero by definition. Inserting the momentum $p_{\mathrm{MP}}$ from Eq. (23) into Eq. (22) and setting $v=v_{\mathrm{g}}=c / n_{\mathrm{g}}$ and $p_{\mathrm{MP}}^{\prime}=0$, we obtain

$$
\delta m=\left(n_{\mathrm{p}} n_{\mathrm{g}}-1\right) \hbar \omega_{0} / c^{2} .
$$

As the final outcome, we have obtained unique values for $p_{\text {MP }}$ and $\delta m$, given in Eqs. (23) and (24). With the Lorentz transformation in Eqs. (21) and (22), these values can be used to unambiguously calculate the total energy and momentum of the MP in arbitrary inertial frames. Therefore, the MP 
quasiparticle is the only model of a light quantum in a medium that fully satisfies the Lorentz transformation and is consistent with the phase and group velocities.

According to the special theory of relativity, we can write the total energy of the MP in the $R$ frame as $m_{0} c^{2}$, where $m_{0}$ is the rest mass of the structural system of the MP. Therefore, inserting $p_{\mathrm{MP}}$ and $\delta m$ from Eqs. (23) and (24) into Eq. (21) together with $v=v_{\mathrm{g}}=c / n_{\mathrm{g}}$, we obtain in the $R$ frame

$$
m_{0}=n_{\mathrm{p}} \sqrt{n_{\mathrm{g}}^{2}-1} \hbar \omega_{0} / c^{2} .
$$

The corresponding MP energy and momentum in the $L$ frame are then given by

$$
\begin{gathered}
E_{\mathrm{MP}}=\gamma_{v_{\mathrm{g}}} m_{0} c^{2}=n_{\mathrm{p}} n_{\mathrm{g}} \hbar \omega_{0}, \\
p_{\mathrm{MP}}=\gamma_{v_{\mathrm{g}}} m_{0} v_{\mathrm{g}}=\frac{n_{\mathrm{p}} \hbar \omega_{0}}{c} .
\end{gathered}
$$

These results essentially generalize the results of Ref. [1] for dispersive media. The energy and momentum in Eq. (26) and the rest mass in Eq. (25) fulfill the covariance condition $E_{\mathrm{MP}}^{2}-\left(p_{\mathrm{MP}} c\right)^{2}=\left(m_{0} c^{2}\right)^{2}$. Although knowing $\delta m$ is enough to understand the mass transfer associated with the MP, $m_{0}$ is useful for transparent understanding of the covariant MP state of light in a medium.

Using the covariant energy-momentum ratio $E / p=c^{2} / v_{\mathrm{g}}$, we can split the total MP momentum in Eq. (26) into parts corresponding to the electromagnetic energy $E_{\text {field }}=\hbar \omega_{0}$ and the MDW energy $E_{\mathrm{MDW}}=\delta m c^{2}$. As a result, we obtain the field's and MDW's shares of the total MP momentaum in the $L$ frame as

$$
\begin{aligned}
p_{\mathrm{MDW}} & =\delta m v_{\mathrm{g}}=\left(n_{\mathrm{p}}-\frac{1}{n_{\mathrm{g}}}\right) \frac{\hbar \omega_{0}}{c}, \\
p_{\text {field }} & =p_{\mathrm{MP}}-p_{\mathrm{MDW}}=\frac{\hbar \omega_{0} v_{\mathrm{g}}}{c^{2}}=\frac{\hbar \omega_{0}}{n_{\mathrm{g}} c} .
\end{aligned}
$$

The field's share of the momentum is of the Abraham form and the MDW's share of the momentum corresponds to the difference of the Minkowski and Abraham momenta.

Using Eqs. (23)-(26) one can easily show that the constant center of energy velocity (CEV) law, essentially equal to Newton's first law, is fulfilled by the MP theory also in the case of dispersive media. We apply the conservation of momentum at the interface where the photon enters a medium block. The photon momentum in vacuum must then be equal to the sum of the MP momentum and the possible recoil momentum received by a thin interface layer of the medium block. We can write the momentum conservation law as $\hbar \omega_{0} / c=p_{\mathrm{MP}}+M_{\mathrm{r}} V_{\mathrm{r}}$, where $M_{\mathrm{r}}=M-\delta m$ is the mass of the medium block from which the mass transferred by the MP has been subtracted. The center of energy velocity $V_{\mathrm{r}}$ of $M_{\mathrm{r}}$ can then be solved from the momentum conservation law as $V_{\mathrm{r}}=\left(1-n_{\mathrm{p}}\right) \hbar \omega_{0} /\left(M_{\mathrm{r}} c\right)$, where we have used the transferred mass given in Eq. (24). Writing the energy of the MP using its rest mass given in Eq. (25) and observing that the atomic velocities are certainly nonrelativistic, we can write the CEV law before and after the photon has entered the medium as

$$
V_{\mathrm{CEV}}=\frac{\sum_{i} E_{i} v_{i}}{\sum_{i} E_{i}}=\frac{\hbar \omega_{0} c}{\hbar \omega_{0}+M c^{2}}=\frac{\gamma m_{0} c^{2} v_{\mathrm{g}}+M_{\mathrm{r}} c^{2} V_{\mathrm{r}}}{\gamma m_{0} c^{2}+M_{\mathrm{r}} c^{2}}
$$

Here the summation is over all material particles and field quanta and $E_{i}$ and $v_{i}$ are their energies and velocities. The equality of the numerators divided by $c^{2}$ corresponds to the momentum conservation and the equality of the denominators is nothing but the conservation of energy. Therefore, Eq. (28) directly shows that the MP model obeys the constant CEV motion in dispersive media, thus generalizing the result derived for nondispersive media in Ref. [1]. This also explains why the derivations of the Minkowski momentum assuming zero rest mass for the light quantum in a medium lead to violation of the constant CEV motion [6,7].

As a side product, by substituting $p_{\mathrm{MP}}$ from Eq. (23) into the first term of Eq. (21), $\hbar \omega_{0}^{\prime}=\gamma_{v}\left(\hbar \omega_{0}-v p_{\mathrm{MP}}\right)$, we obtain $\hbar \omega_{0}^{\prime}=\gamma_{v}\left(1-n_{\mathrm{p}} v / c\right) \hbar \omega_{0}$, which is the well-known Doppler-shifted energy of a photon in a medium in an arbitrary frame moving with the velocity $v$ with respect to the $L$ frame [37]. Thus, the total MP momentum of the Minkowski form in Eq. (23) can also be derived from the Doppler shift [6,38], which, however, must be used as a part of the Lorentz transformation in Eqs. (21) and (22) in order to enable the determination of the transferred mass $\delta m$ of the MP.

\section{OCD SIMULATIONS}

Above, we have derived the MP theory for dispersive media using the complementary MP quasiparticle and OCD models. In order to show the correspondence between these models and to illustrate the MDW and the actual atomic displacements due to optoelastic forces in dispersive media, we present numerical OCD simulations of a Gaussian light pulse propagating in linearly and nonlinearly dispersive sample media. To facilitate the planning of possible experiments, we also compute the atomic displacements due to the MDW in silicon.

\section{A. Visualization of the node structure of the MDW}

\section{Linearly dispersive medium}

First, we apply the OCD model to illustrate the node structure of the MDW and the actual atomic displacements due to a Gaussian light pulse in a linearly dispersive material. The Gaussian light pulse of Eq. (5) is assumed to have a vacuum wavelength of $\lambda_{0}=1550 \mathrm{~nm}$ and a total electromagnetic energy of $U_{0}=1 \mu \mathrm{J}$. We assume that the relative spectral width of the pulse, in our example, is $\Delta \omega / \omega_{0}=\Delta k_{0} / k_{0}=$ 0.05 corresponding to $\Delta t_{\mathrm{FWHM}}=27 \mathrm{fs}$. The FWHM is fixed to this close to feasibility limit value to make the node structure of the MDW visible. In our simulations, we use space discretization of $h_{x}=\lambda / 40$ and time discretization of $h_{t}=2 \pi /\left(40 \omega_{0}\right)$ that are sufficiently dense compared to the scale of the harmonic cycle. The computational details of the simulation are described in Appendix C of Ref. [1].

For visualization needs, we use here an artificial example material for which the refractive indices at the central frequency $\omega_{0}=2 \pi c / \lambda_{0}$ are $n_{\mathrm{p}}=1.5$ and $n_{\mathrm{g}}=2$. The chosen phase refractive index is close to typical values for glasses but we have made the group refractive index somewhat larger to enable the visual separation of the phase velocity dynamics of the nodes inside the Gaussian envelope. The diameter $d$ of the circular cross-sectional area $A=\pi(d / 2)^{2}$ is assumed to be $d=100 \mu \mathrm{m}$, which is chosen to be large enough so that the 

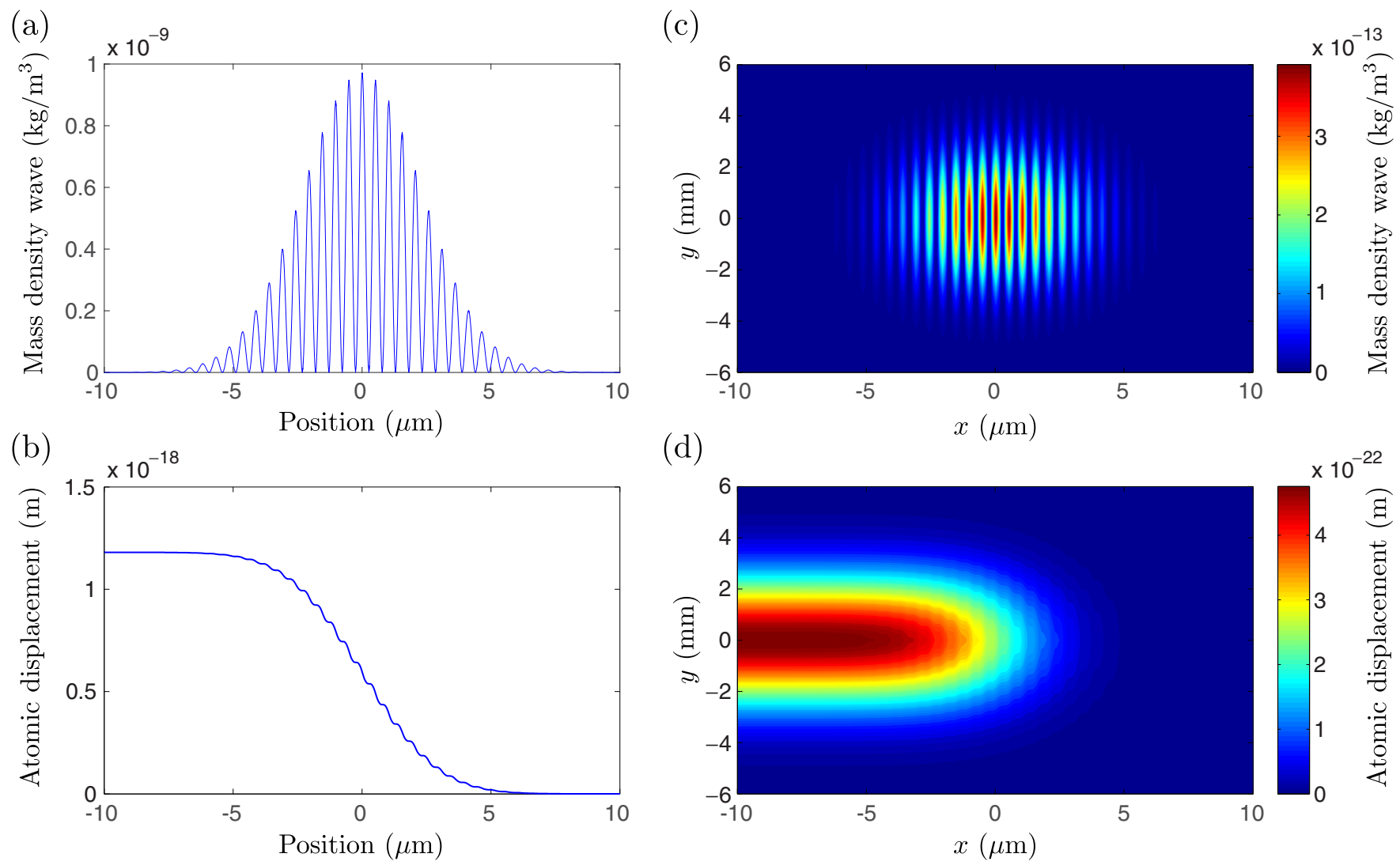

FIG. 4. Illustration of (a) the MDW and (b) the atomic displacements in a linearly dispersive material where the phase and group refractive indices for the central frequency are $n_{\mathrm{p}}=1.5$ and $n_{\mathrm{g}}=2$. The Gaussian light pulse has a vacuum wavelength $\lambda_{0}=1550 \mathrm{~nm}, \Delta t_{\mathrm{FWHM}}=27 \mathrm{fs}$, and energy $U_{0}=1 \mu \mathrm{J}$ per cross-sectional area of diameter $d=100 \mu \mathrm{m}$. The pulse parameters are close to technological feasibility limit but they are chosen to visualize the node structure of the MDW. Panel (c) shows the MDW and (d) shows the atomic displacements of a three-dimensional light pulse with finite lateral dimensions in the plane $z=0 \mathrm{~m}$.

resulting maximum value $3.3 \times 10^{11} \mathrm{~W} / \mathrm{cm}^{2}$ of the Poynting vector averaged over the harmonic cycle is below the bulk value of the breakdown threshold irradiance of many common materials, e.g., $5.0 \times 10^{11}$ reported for fused silica [39]. The equilibrium density of the material is assumed to be $\rho_{0}=2400$ $\mathrm{kg} / \mathrm{m}^{3}$, the material is assumed to be isotropic, and we use the value $B=50 \mathrm{GPa}$ for the bulk modulus and $G=25 \mathrm{GPa}$ for the shear modulus. These values are close to typical values of the corresponding quantities for glass.

Figure 4(a) shows the simulated MDW as a function of position when the light pulse is propagating at the position $x=0 \mu \mathrm{m}$. The time-dependent simulation is presented as a video file in the Supplemental Material [40]. The MDW equals the difference of the disturbed mass density $\rho_{\mathrm{a}}(\mathbf{r}, t)$ and the equilibrium mass density $\rho_{0}$ of the medium and it is obtained by solving Newton's equation of motion in Eq. (10). The MDW is driven by the optoelastic forces due to the Gaussian light pulse. The envelope of the MDW clearly follows the Gaussian form of the pulse as expected. As the light pulse is not very long compared to the harmonic cycle, the node structure of the MDW can be seen in the same scale with the Gaussian envelope. When we integrate the MDW mass density in Fig. 4(a), we obtain the total transferred mass of $2.23 \times 10^{-23} \mathrm{~kg}$. Dividing this by the photon number of the light pulse, we then obtain the value of $1.60 \mathrm{eV} / \mathrm{c}^{2}$ for the transferred mass per photon. Within the relative error of $10^{-4}$, this equals the MP quasiparticle value obtained from Eq. (24). For a more detailed discussion of the correspondence between the MP quasiparticle and OCD approaches, see Sec. VII.

Figure 4(b) shows the atomic displacements corresponding to MDW in Fig. 4(a), again, as a function of position. On the left of the light pulse, the atomic displacement has a constant value of $r_{\mathrm{a}, \max }=1.18 \times 10^{-18} \mathrm{~m}$. This follows from the optical force in the second term of Eq. (9). Within the relative error of $10^{-4}$, we obtain $r_{\mathrm{a} \text {, max }}=\delta M /\left(\rho_{0} A\right)$, where $\delta M=N_{0} \delta m$ is the total transferred mass of the light pulse. The leading edge of the optical pulse is propagating to the right approximately at the position $x=7 \mu \mathrm{m}$. Therefore, to the right of $x=7 \mu \mathrm{m}$, the atomic displacement is zero. The optoelastically driven MDW is manifested by the fact that atoms are more densely spaced at the position of the light pulse as the atoms on the left of the pulse have been displaced forward and the atoms on the right of the pulse are still at their equilibrium positions. The momentum of atoms in the MDW is obtained by integrating the classical momentum density as given in Eq. (18) at an arbitrary time.

We also illustrate the MDW and the atomic displacements due to a three-dimensional light pulse. This light pulse is only an approximative solution of Maxwell's equations. It is obtained from the one-dimensional pulse described by Eq. (5) by adding additional $y$ and $z$ dependencies by using factors $e^{-\left(\Delta k_{y}\right)^{2} y^{2} / 2}$ and $e^{-\left(\Delta k_{z}\right)^{2} z^{2} / 2}$. As reasoned in Ref. [1], 
this approximation becomes accurate if $\Delta k_{y}$ and $\Delta k_{z}$ are sufficiently small compared to the wave number of the central frequency in the medium equal to $k_{0, \text { med }}=n_{\mathrm{p}} k_{0}$. In our example, we use $\Delta k_{y}=\Delta k_{z}=10^{-4} k_{0}$, which are small so that the approximation is well justified for our visualization purposes.

The contour plot in Fig. 4(c) shows the MDW of the three-dimensional Gaussian pulse in the plane $z=0 \mathrm{~m}$. The corresponding time-dependent simulation is presented as a video file in the Supplemental Material [40]. The threedimensional pulse differs from the one-dimensional pulse in Fig. 4(a) by its finite lateral dimensions as described above. The values of the MDW in Fig. 4(c) are thus smaller than the values in Fig. 4(a) due to the smaller value of the energy per cross-sectional area.

The contour plot in Fig. 4(d) presents the $x$ component of the atomic displacements due to the three-dimensional Gaussian pulse in the plane $z=0 \mathrm{~m}$. The values of the atomic displacement in Fig. 4(d) are smaller than the values in the one-dimensional case in Fig. 4(b), again, due to the smaller value of the energy per cross-sectional area.

\section{Nonlinearly dispersive medium}

Next we investigate the MDW in a nonlinearly dispersive example material. The nonlinear dispersion is described by the simple Lorentz model of a dielectric in Sec. III C. We use the same parameters for the Gaussian pulse as above. The only difference is the use of the nonlinear dispersion relation in Eq. (7), in which the model parameters $\omega_{\mathrm{r}}=1.63299 \omega_{0}$ and $\omega_{\mathrm{p}}=1.44338 \omega_{0}$ have been determined so that the phase and group refractive indices have the same values $n_{\mathrm{p}}=1.5$ and $n_{\mathrm{g}}=2$ for the central frequency of the pulse as above. As $\omega_{0}<\omega_{\mathrm{r}}$, the dispersion relation corresponds to the lower polariton branch in Fig. 3 .

We start by briefly discussing how the nonlinear dispersion relation affects the pulse shape in comparison with the linear dispersion relation. At $t=0 \mathrm{fs}$, the electric field of the pulse is determined by its Fourier components that are chosen to be of the same Gaussian form as in the case of linear dispersion above. In dispersive media, the Fourier components of the magnetic field are given by $\tilde{H}(k)=Z(k) \tilde{E}(k)$, where the proportionality factor $Z(k)=\sqrt{\mu[\omega(k)] / \varepsilon[\omega(k)]}$ is the $k$-dependent wave impedance. Due to this $k$-dependent proportionality factor, the Fourier components of the magnetic field and the resulting pulse shapes are modified depending on the dispersion relation even at $t=0 \mathrm{fs}$. However, for light pulses with a narrow spectral width, the deviation in the pulse shape between the nonlinear and the corresponding linear dispersion relation is typically very small for $t=0 \mathrm{fs}$. At later times $t>0 \mathrm{fs}$, the dispersion-modified time dependence through the exponential factor $e^{-i \omega(k) t}$ of the fields in Eqs. (1) and (2) more clearly affects the pulse shape. Therefore, in order to illustrate the effect of nonlinear dispersion, we compare the MDW pulse shapes at $t=0$ and $t=500 \mathrm{fs}$.

Figure 5(a) presents the MDW of the Gaussian pulse in the nonlinearly dispersive medium at these two instances of time. The corresponding time-dependent simulation is presented as a video file in the Supplemental Material [40]. The pulse on the left in Fig. 5(a) corresponds to the pulse
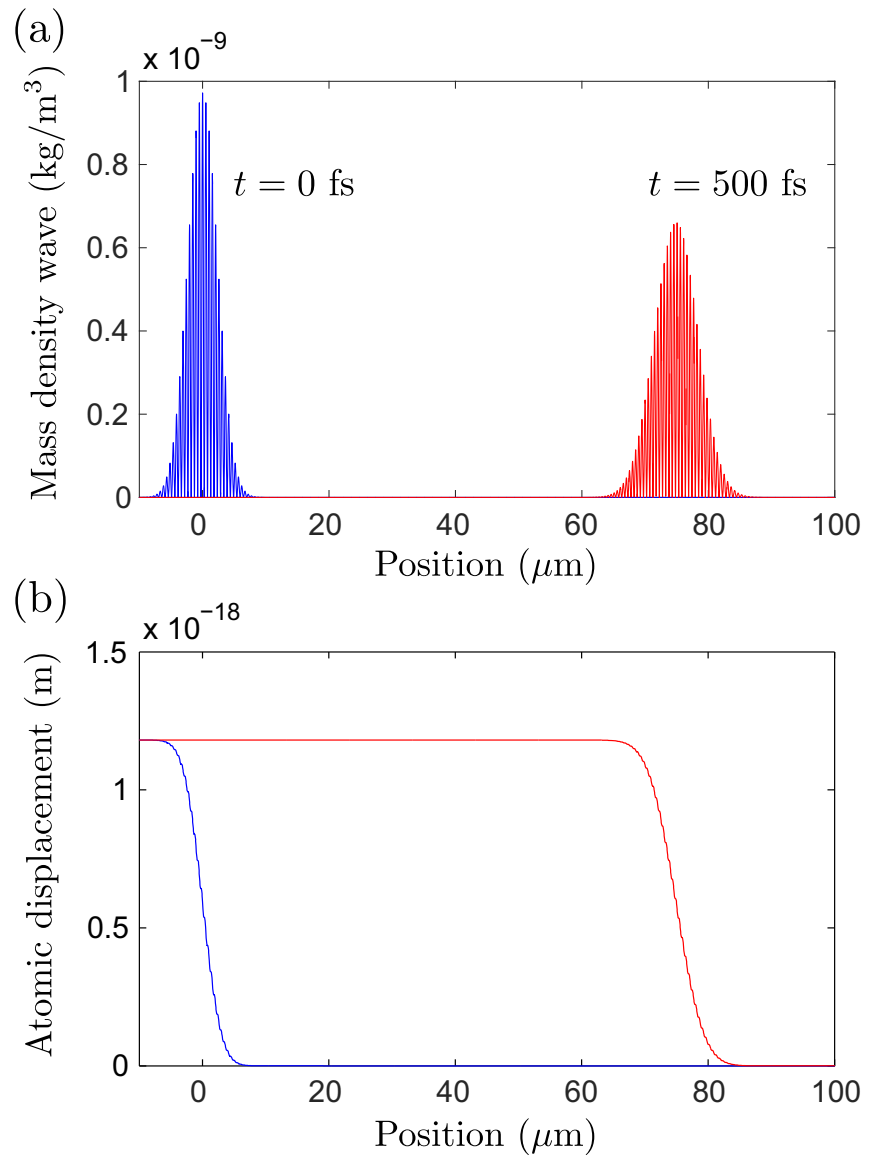

FIG. 5. (a) The MDW and (b) the atomic displacement of a Gaussian light pulse in a nonlinearly dispersive material as a function of the position at two instances of time: $t=0$ fs (blue) and $t=500 \mathrm{fs}$ (red). The vacuum wavelength is $\lambda_{0}=1550 \mathrm{~nm}$ and the corresponding phase and group refractive indices are $n_{\mathrm{p}}=1.5$ and $n_{\mathrm{g}}=2$.

at $t=0$ fs while the pulse on the right corresponds to the pulse at $t=500 \mathrm{fs}$. During this time interval, the pulse has propagated to the right a distance of $75 \mu \mathrm{m}$. One can clearly see that the pulse has become lower and broadened when compared to the initial pulse. This effect follows purely from the nonlinearity of the dispersion relation. If the dispersion relation would be perfectly linear, this broadening would not occur as the Gaussian envelope would maintain its width.

Figure 5(b) shows the atomic displacements corresponding to the MDW in Fig. 5(a) at $t=0$ and $t=500$ fs. The most clear physical difference between the atomic displacements at these two moments of time is that the slope of the atomic displacement curve in Fig. 5(b) is lowered at $t=500 \mathrm{fs}$. This follows from the broadening of the light pulse. The atomic displacements on the left of the pulse are approximatively equal as expected. This constant value, $r_{\mathrm{a}, \max }$, depends on the phase and group refractive indices and the density of the material, but it is only slightly affected by the nonlinearity of the dispersion relation. This can be seen by observing that the constant value of the atomic displacement on the left of the light pulse in Fig. 5(b) is closely equal to the corresponding value in Fig. 4(c). This is related to the fact that we have used the same density for the material and defined the nonlinear 
dispersion relation so that the phase and group refractive indices for the central frequency in our nonlinear case are equal to the same quantities in the case of linear dispersion above.

\section{Continuous wave}

By changing the Gaussian light pulse to a top-hat pulse and by making the length of the pulse very large, we can also use the time-dependent OCD model to simulate a continuous-wave (cw) laser beam. The cw beam deserves a separate comment since it has been extensively discussed in previous theoretical works and in the analysis of experiments. Previous theoretical works have often concluded that, since the time average of the Abraham force given by the second term of Eq. (9) is zero for the cw field, its effect is not directly observable [30]. This conclusion is not sound. The maxima of the cw field energy give rise to alternative acceleration and deceleration of the atoms in the medium in the direction of the light beam. As a net effect, the atoms are displaced in the direction of the beam and, in the average, they also carry momentum. This changes the dynamical state of the medium and also leads to shift of the atomic density which must be accounted for in the analysis of experiments. Note that in the simulations of the dynamical state of the medium using the OCD method, whether we analyze a light pulse in a solid using elasticity theory or in a liquid using Navier-Stokes equation, we cannot assume that the medium is incompressible, which is often done [8]. In a perfectly incompressible medium, the medium dynamics cannot follow the time and position dependence of the electromagnetic field in a way governed by Newton's equation of motion in Eq. (10).

In long time scales, the elastic forces that try to restore the mass equilibrium in the medium also play an important role. Assuming the geometry of a medium block whose transverse boundaries are held fixed by external forces, the OCD model leads to an equilibrium where the forward mass transfer due to the MDW is balanced by the backward mass transfer due to elastic waves. The accumulation of the elastic waves together with the absorption of photons also lead to heating of the medium block. Therefore, in order to obtain an equilibrium in the simulation, one must also account for the transport of the generated heat over the boundaries of the medium block by conduction and radiation. The detailed study of this cw case is left as a topic of further work.

\section{B. Estimating atomic displacements of the MDW in silicon}

Next we study how the atomic displacement of the MDW depends on the pulse energy and the diameter of the crosssectional area. These calculations are presented for designing experimental setups for the measurement of the transferred mass of a light pulse. We simulate a one-dimensional Gaussian pulse in silicon for different pulse energies, cross-sectional areas, and $\Delta t_{\mathrm{FWHM}}$. The computed atomic displacements correspond to the experimental arrangement in which the given pulse energy is propagating in a waveguide or an optical fiber as schematically illustrated in Fig. 6. Due to the interface effects, the cross-sectional area of the fiber cannot be directly compared with the cross-sectional area of our calculations. The core cross section of the waveguide or fiber should be corrected for the possible cladding layer, metallic coating, and other

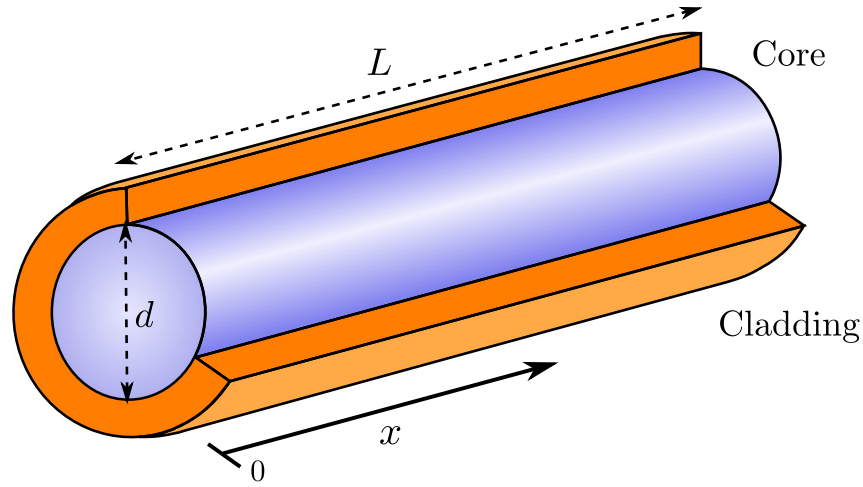

FIG. 6. Schematic illustration of a waveguide or an optical fiber with a core diameter $d$ and length $L$. The transferred mass of the MDW is to be measured as the shift of atoms on the surface of the waveguide at $x=L / 2$ just after the light pulse has gone.

factors that influence the spreading of the pulse energy in the transverse direction. In detailed calculations, the waveguide dispersion should also be taken into account. All these factors can be easily accounted for in the OCD simulations.

The phase and group refractive indices of silicon are given by $n_{\mathrm{p}}=3.4757$ and $n_{\mathrm{g}}=3.5997$ for $\lambda_{0}=1550 \mathrm{~nm}$ [41]. The density is $\rho_{0}=2329 \mathrm{~kg} / \mathrm{m}^{3}$ [42] and the elastic constants in the direction of the (100) plane are $C_{11}=165.7 \mathrm{GPa}, C_{12}=$ 63.9 GPa, and $C_{44}=79.6 \mathrm{GPa}$ [43]. These elastic constants correspond to the bulk modulus of $B=\left(C_{11}+2 C_{12}\right) / 3=$ $97.8 \mathrm{GPa}$ and the shear modulus of $G=C_{44}=79.6 \mathrm{GPa}$.

Figure 7 shows the atomic displacement as a function of the pulse energy and the diameter of the cross-sectional area. Compared to the femtosecond pulses above, we here assume longer pulses with $\Delta t_{\mathrm{FWHM}}>1 \mathrm{~ns}$. Therefore, the correspondence of the MP quasiparticle and the OCD models is very accurate and we can use the quasiparticle model result $r_{\text {a,max }}=\delta M /\left(\rho_{0} A\right)$ for the maximum atomic displacement $r_{\mathrm{a}, \max }$. Using $\delta M=\left(n_{\mathrm{p}} n_{\mathrm{g}}-1\right) U_{0} / c^{2}$ and $A=\pi(d / 2)^{2}$, where $d$ is the diameter of the cross-sectional area, we

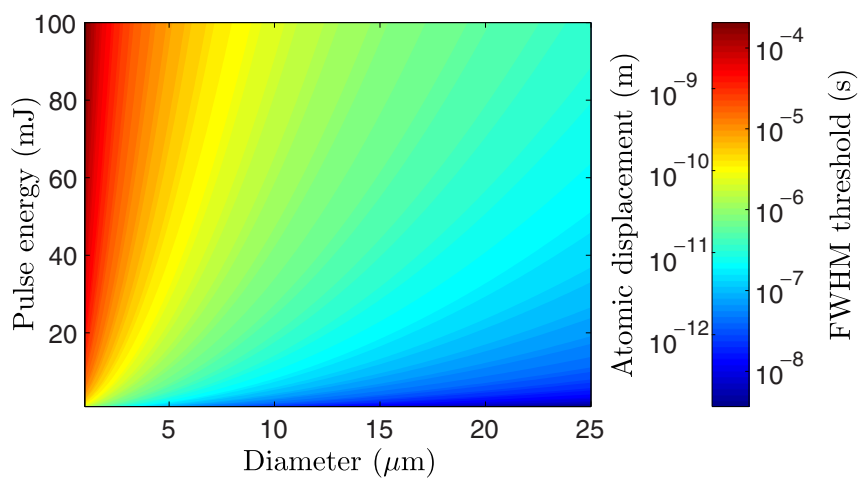

FIG. 7. The total atomic displacement of the MDW of a Gaussian light pulse in silicon as a function of the pulse energy and the diameter of the cross-sectional area of the pulse. The vacuum wavelength is $\lambda_{0}=1550 \mathrm{~nm}$ and the corresponding phase and group refractive indices are $n_{\mathrm{p}}=3.4757$ and $n_{\mathrm{g}}=3.5997$. The second color-bar axis shows the threshold $\Delta t_{\text {th }}$ of the pulse obtained by requiring that the bulk value of the breakdown threshold irradiance of the material is not exceeded. 
then obtain $r_{\mathrm{a} \text {, max }}=\left(n_{\mathrm{p}} n_{\mathrm{g}}-1\right) U_{0} /\left[c^{2} \rho_{0} \pi(d / 2)^{2}\right]$. Hence the atomic displacement depends linearly on the pulse energy while it is inversely proportional to the cross-sectional area. Consequently, in Fig. 7, the atomic displacement is seen to be large for high pulse energies and for small cross-sectional areas as expected.

\section{Influence of the material breakdown irradiance}

We also evaluate the minimum $\Delta t_{\text {FWHM }}$ of a Gaussian pulse that is needed to produce the corresponding atomic displacement without exceeding the bulk value of the breakdown threshold irradiance of the material. Using the total electromagnetic energy of the pulse given by $U_{0}$, and the cross-sectional area of the pulse given by $A=\pi(d / 2)^{2}$, this threshold $\Delta t_{\mathrm{FWHM}}$, denoted by $\Delta t_{\mathrm{th}}$, is calculated as $\Delta t_{\text {th }}=$ $2 U_{0} /\left[\pi(d / 2)^{2} I_{\mathrm{th}}\right]$, where $I_{\mathrm{th}}$ is the bulk value of the breakdown threshold irradiance of the material. The corresponding fluence is $F_{\text {th }}=2 U_{0} /\left[\pi(d / 2)^{2}\right]$. The factor 2 comes from the fact that the pulse is Gaussian and not a top-hat pulse with constant irradiance. For silicon with $\lambda_{0}=1550 \mathrm{~nm}$, the bulk value of the breakdown threshold energy density has been reported to be $u_{\text {th }}=13.3 \mathrm{~J} / \mathrm{cm}^{3}$ [44], which corresponds to the threshold irradiance of $I_{\mathrm{th}}=u_{\mathrm{th}} v_{\mathrm{g}}=1.11 \times 10^{11} \mathrm{~W} / \mathrm{cm}^{2}$. These are values averaged over the harmonic cycle.

The threshold $\Delta t_{\text {th }}$ of a Gaussian pulse calculated as explained above is presented by the second color-bar axis in Fig. 7. Using the relations above, the scaling between the atomic displacement and the threshold $\Delta t_{\text {th }}$ is given by $r_{\mathrm{a}, \max } / \Delta t_{\mathrm{th}}=\left(n_{\mathrm{p}} n_{\mathrm{g}}-1\right) I_{\mathrm{th}} /\left(2 c^{2} \rho_{0}\right)$. This clearly indicates that, in order to obtain large atomic displacements for a given pulse energy, it is beneficial to have a material with a high refractive index, high breakdown threshold irradiance, and relatively small mass density. In Fig. 7, one can see that, in order to obtain atomic displacements larger than $1 \mathrm{~nm}$ in silicon without breaking the material, the pulse width must be larger than $\Delta t_{\text {th }}=33 \mu \mathrm{s}$.

\section{Displacement of atoms due to optical absorption}

In measuring the atomic displacements due to the MDW, one essential point is to ensure that the momentum transfer due to optical absorption of the material is not too large so that the resulting atomic movement would exceed the photon mass drag effect. Therefore, we estimate the atomic displacement and the atomic velocity resulting from the optical absorption. The mass of a cylindrical medium block with a diameter $d$ and length $L$, or the core of the waveguide in Fig. 6, is given by $M=\rho_{0} \pi(d / 2)^{2} L$. The momentum absorbed by this medium block is given by $P_{\mathrm{abs}}=\left(1-e^{-\alpha L}\right) n_{\mathrm{p}} U_{0} / c \approx$ $\alpha L n_{\mathrm{p}} U_{0} / c$, where $\alpha$ is the small absorption coefficient of the medium. The velocity obtained by the medium block is then $V_{\mathrm{abs}}=P_{\mathrm{abs}} / M \approx \alpha n_{\mathrm{p}} U_{0} /\left[c \rho_{0} \pi(d / 2)^{2}\right]$. In the time scale of $\Delta t_{\mathrm{FWHM}}$, the resulting atomic displacement is given by $X_{\mathrm{abs}}=V_{\mathrm{abs}} \Delta t_{\mathrm{FWHM}}$.

In the case of silicon, absorption is very low at $\lambda_{0}=1550$ nm. The measurements by Schinke et al. [45] and Green [46] for $\lambda_{0}=1450 \mathrm{~nm}$ give $\alpha \approx 10^{-8} \mathrm{~cm}^{-1}$ and the absorption is known to decrease towards $\lambda_{0}=1550 \mathrm{~nm}$. Therefore, we can conservatively estimate $\alpha=10^{-8} \mathrm{~cm}^{-1}$. Using $\Delta t_{\mathrm{FWHM}}=$ $\Delta t_{\mathrm{th}}=33 \mu \mathrm{s}$ and $d=2.5 \mu \mathrm{m}$ corresponding to $r_{\mathrm{a}, \max }=$
$1.0 \mathrm{~nm}$ atomic displacement due to the MDW, and solving the threshold pulse energy from $\Delta t_{\text {th }}=2 U_{0} /\left[\pi(d / 2)^{2} I_{\text {th }}\right]$, we obtain $U_{0}=90 \mathrm{~mJ}$. The velocity of atoms is then $V_{\mathrm{abs}}=9.1 \times$ $10^{-8} \mathrm{~m} / \mathrm{s}$ and, in the time scale of $\Delta t_{\mathrm{th}}$, the resulting atomic displacement is given by $X_{\mathrm{abs}}=3.0 \mathrm{pm}$. This atomic displacement due to optical absorption is clearly smaller than $r_{\mathrm{a}, \max }=$ $1.0 \mathrm{~nm}$ following from the photon mass drag effect. Therefore, optical absorption is not expected to prevent measurements of the atomic displacements due to the photon mass drag effect. This result strongly supports the experimental feasibility of the measurement of the transferred mass of the MDW.

We have also considered the thermal expansion following from the optical absorption. Using the well-known specificheat capacity and thermal-expansion coefficients, it can be shown that the thermal expansion does not lead to measurable atomic displacements in the middle part of the fiber in the time scale of $\Delta t_{\text {FWHM }}$ that is shorter than the time that it takes for sound waves to travel through the fiber. This is also related to the longitudinal relaxation studied below.

\section{Transverse relaxation}

In the experimental verification of the transferred mass of the MDW, one also has to account for the phonon relaxation of the atomic displacements due to the MDW. This relaxation takes place at the velocity of sound and it is governed by Eqs. (12)-(14) of the OCD model. The relaxation effect has been briefly studied in Ref. [1]. If a three-dimensional light pulse propagates inside a medium or in the core of an optical fiber that has a cladding, the MDW displaces atoms as shown in Fig. 4(d). The atoms along the path of the MP are displaced forward while the atoms in the surrounding layers are not shifted. This results in a shear strain field along the path of the MP. The transverse relaxation refers to the relaxation of the strain field so that atoms in the displaced region are shifted backwards and atoms in the surrounding layers are shifted forwards. After the transverse relaxation, the longitudinal strain becomes constant across the cross section of the waveguide.

The relaxation of the strain field is quite fast in optical fibers where the distances to be traveled by phonons in the transverse direction are very short. Using the longitudinal velocity of sound in silicon, given by $v_{\|}=\sqrt{C_{11} / \rho_{0}}=8435$ $\mathrm{m} / \mathrm{s}$, the time at which a sound wave propagates, e.g., a distance of $1 \mathrm{~mm}$ is $1.2 \mathrm{~ns}$. This is obviously very short compared to the time scale of $\Delta t_{\mathrm{FWHM}}=33 \mu \mathrm{s}$ used above. Thus, as a net effect, this transverse relaxation takes place in a time scale that is shorter than the passing of the pulse and we can approximate that, in a narrow waveguide, atoms are displaced in the longitudinal direction by the same amount in the middle and at the surface of the waveguide. After the transverse relaxation, the constant atomic displacement is reduced to $r_{\mathrm{a} \text {,relaxed }}=r_{\mathrm{a} \text {, max }} \rho_{0} A /\left(\rho_{\mathrm{eff}} A_{\mathrm{tot}}\right)$, where $A_{\text {tot }}$ is the total cross-sectional area of all layers and $\rho_{\text {eff }}$ is the effective mass density of the cross-sectional area given by $\rho_{\text {eff }}=\sum_{i} \rho_{i} A_{i} / A_{\text {tot }}$, where the sum is taken over all material layers and $\rho_{i}$ and $A_{i}$ are the densities and cross-sectional areas of the corresponding layers.

The time constant of the transverse relaxation is much shorter than the pulse width $\Delta t_{\mathrm{FWHM}}$ for structures where the atomic displacement is potentially measurable. Therefore, it 
is advantageous to keep the waveguide diameter as small as possible considering the effectivity of the coupling of the light source to the waveguide and the technical processing aspects of fabricating it. This suggests that the narrower the waveguide is, the larger is the atomic displacement and the breakdown of the material can be prevented by increasing the pulse width $\Delta t_{\text {FWHM }}$. However, the longitudinal relaxation described in the next subsection will set a limit for increasing $\Delta t_{\mathrm{FWHM}}$.

\section{Longitudinal relaxation}

After the transverse relaxation has taken place, further relaxation can only occur starting from the ends of the fiber, which have experienced recoil effects and which may be attached to some part of the experimental setup that tries to keep them fixed. If the fiber is long enough, these longitudinal relaxation waves starting from the ends of the fiber have not time enough to reach the middle part of the fiber where the atomic displacement is to be measured. The distance traveled by sound in silicon in the time scale of $\Delta t_{\mathrm{FWHM}}=33 \mu \mathrm{s}$ is $28 \mathrm{~cm}$. Therefore, a fiber with length $L>56 \mathrm{~cm}$ is sufficient to avoid the longitudinal relaxation from having an effect on the measured value of the atomic displacement assuming that the atomic displacement in the middle part of the fiber is measured just after the light pulse has gone.

\section{DISCUSSION AND COMPARISON OF THE MP AND OCD RESULTS}

\section{A. Dependence on the pulse width}

We have shown in Ref. [1] that, in the case of nondispersive media, the MP and OCD models give equal results within the relative numerical accuracy of the OCD simulations. For a dispersive medium, the comparison of the MP and OCD momenta becomes more subtle. The derivation of the MP model in Sec. V assumes infinitely narrow pulse in the frequency domain while the OCD model involves integration over partial waves and thus accounts for the frequency-dependent dispersion relation. Using Eqs. (15)-(20) and (23)-(27), the total transferred mass and the total momentum and the momentum of the field and the MDW can be written for a dispersive medium as given in Table I.

In order to study the correspondence between the MP and OCD results in a dispersive medium, we plot the relative difference in the total momentum $\mathbf{P}_{\mathrm{MP}}$ obtained from the MP and OCD models as a function of the relative spectral width $\Delta \omega / \omega_{0}$ of a Gaussian light pulse. The relative difference is

TABLE I. The transferred mass, the total momentum, the field's share of the momentum, and the MDW's share of the momentum calculated by using the MP and OCD models. Here $N_{0}=U_{0} / \hbar \omega_{0}$ is the photon number of the pulse.

\begin{tabular}{lcc}
\hline \hline & OCD & MP \\
\hline$\delta M$ & $\int \rho_{\mathrm{MDW}} d^{3} r$ & $\left(n_{\mathrm{p}} n_{\mathrm{g}}-1\right) \frac{N_{0} \hbar \omega_{0}}{c^{2}}$ \\
$\mathbf{P}_{\mathrm{MP}}$ & $\int\left(\rho_{\mathrm{a}} \mathbf{v}_{\mathrm{a}}+\frac{\mathbf{E} \times \mathbf{H}}{c^{2}}\right) d^{3} r$ & $\frac{n_{\mathrm{p}} N_{0} \hbar \omega_{0}}{c} \hat{\mathbf{x}}$ \\
$\mathbf{P}_{\text {field }}$ & $\int \frac{\mathbf{E} \times \mathbf{H}}{c^{2}} d^{3} r$ & $\frac{N_{0} \hbar \omega_{0}}{n_{\mathrm{g}} c} \hat{\mathbf{x}}$ \\
$\mathbf{P}_{\mathrm{MDW}}$ & $\int \rho_{\mathrm{a}} \mathbf{v}_{\mathrm{a}} d^{3} r$ & $\left(n_{\mathrm{p}}-\frac{1}{n_{\mathrm{g}}}\right) \frac{N_{0} \hbar \omega_{0}}{c} \hat{\mathbf{x}}$ \\
\hline \hline
\end{tabular}

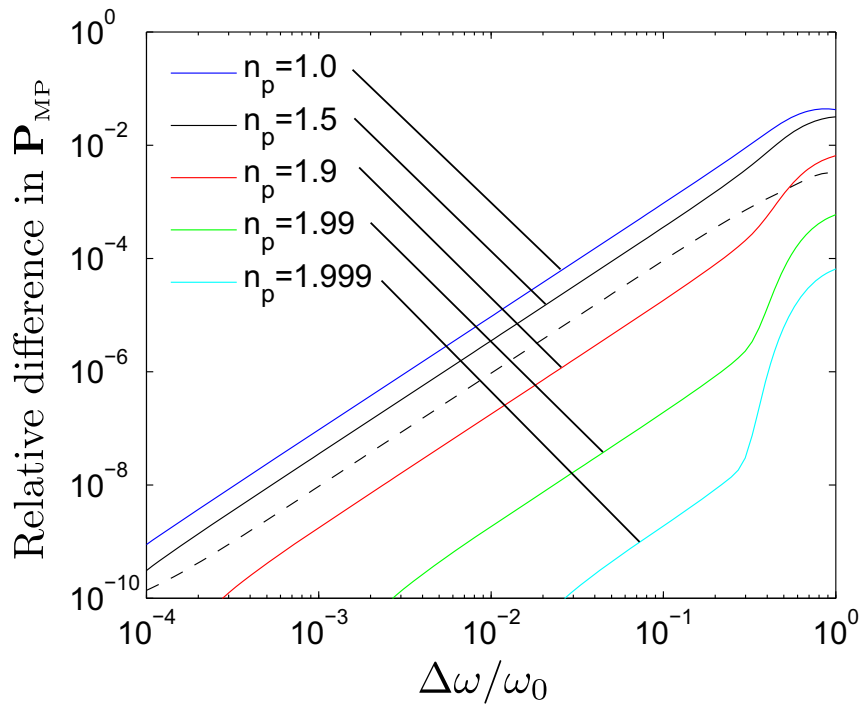

FIG. 8. Relative difference in $\mathbf{P}_{\mathrm{MP}}$ obtained from the MP and OCD models as a function of the relative spectral width of a Gaussian light pulse. The wavelength is fixed to $\lambda_{0}=1550 \mathrm{~nm}$ and the group refractive index is fixed to $n_{\mathrm{g}}=2$. Solid lines show the relative difference calculated by using the linear dispersion relation for selected values of the phase refractive index. The dashed line shows the corresponding plot assuming the nonlinearly dispersive material studied in Sec. VIA, where $n_{\mathrm{p}}=1.5$ and $n_{\mathrm{g}}=2$ for the central frequency.

plotted in Fig. 8 for selected values of the phase refractive index of a linearly dispersive material when the group refractive index is fixed to $n_{\mathrm{g}}=2$ and $\lambda_{0}=1550 \mathrm{~nm}$.

Figure 8 shows that, for dispersive media, the OCD and MP momenta are not equal but their relative difference depends critically on the pulse width. For dispersive media, the MP and OCD results become equal only in the narrow band limit $\Delta \omega / \omega_{0} \rightarrow 0$ for which the relative difference in $\mathbf{P}_{\mathrm{MP}}$ becomes zero. For relative difference values smaller than $10^{-10}$, the graphs saturate to a constant value (not shown) following from the accuracy of the numerical simulation. It is also found that the relative difference in the transferred mass obtained by using the MP and OCD models behaves the same way as the relative difference in the total momentum in Fig. 8. At the narrow band limit, both models describe very accurately the same electromagnetic pulse. Thus, one may argue, in analogy to the nondispersive case [1], that since the MP and OCD models are representations of the same covariant theory for the singlephoton and continuum fields, respectively, the results predicted by them must be equal.

That the results of the MP and OCD models are not exactly equal for a broader pulse can be understood by looking at the expressions of the electric and magnetic fields of the pulse, see Eqs. (1) and (2), and in particular, keeping in mind the dispersion relation and the related $k$ dependence of the Fourier components $\tilde{E}(k) e^{-i \omega(k) t}$ and $\tilde{H}(k) e^{-i \omega(k) t}$. Thus, the OCD model effectively accounts for the frequency dependence of the refractive index. Accordingly, the OCD result cannot be exactly equal to the MP result if we multiply the MP total momentum by photon number of the pulse and keep the refractive indices fixed to their central frequency values. Note 
also that the accuracy of the total momentum given by the OCD model is limited by the fact that the electromagnetic energy density formula in Eq. (15) used in the OCD model is known to hold exactly only in the limit of a monochromatic field [2]. Applying the OCD model with a more accurate form of the energy density given in Ref. [35] is a topic of further work.

The dashed line in Fig. 8 represents the result for the nonlinearly dispersive case studied in Sec. VIA, where $n_{\mathrm{p}}=$ 1.5 and $n_{\mathrm{g}}=2$ for the central frequency. One can see that this graph is qualitatively similar to the graphs obtained for linearly dispersive media. This indicates that the nonlinearity of the dispersion relation does not significantly influence the relative difference between the MP and OCD results.

\section{B. Comparison of the MP and OCD models with previous experiments and theories}

Neither previous experiments or theories have determined the transferred mass of the light pulse, but we can still compare the total MP and OCD momenta of the light pulse with previous works. In the narrow-band limit, both the MP and OCD models give the total momentum which is of the Minkowski form $p_{\mathrm{MP}}=n_{\mathrm{p}} N_{0} \hbar \omega_{0} / c$. This result is in accordance with the laser beam experiment of Jones and Leslie [21], which as discussed in Sec. II supports the Minkowski formula. The experiments of Jones and Leslie were carried out for transparent liquids for which the relaxation dynamics follows Navier-Stokes equation instead of the elasticity theory. However, the relaxation dynamics has extremely small influence on the total momentum of the light pulse. Thus, our simulations for solids can also explain the results of Jones and Leslie. For a reference of possible future experiments making use of a broad pulse, one should note that the OCD model gives a more accurate total momentum. The conventional definition of the Minkowski momentum $p_{\mathrm{MP}}=n_{\mathrm{p}} N_{0} \hbar \omega_{0} / c$ is not meaningful since, instead of a constant $n_{\mathrm{p}}$, we should use a phase index that is appropriately averaged over the broad band pulse.

Previously, Garrison et al. [36] have encountered into a problem that the single-photon expectation value of the momentum $p_{\mathrm{M}}=\int \mathbf{g}_{\mathrm{M}} d^{3} r$, obtained by using the Minkowski momentum density $\mathbf{g}_{\mathrm{M}}=\mathbf{D} \times \mathbf{B}$, is not equal to the commonly defined Minkowski momentum $\tilde{p}_{\mathrm{M}}=n_{\mathrm{p}} \hbar \omega / c$ following from the de Broglie hypothesis or from the present theory but rather $p_{\mathrm{M}}=n_{\mathrm{p}}^{2} \hbar \omega /\left(n_{\mathrm{g}} c\right)$. This controversy has prompted Barnett to introduce different kinetic and canonical values for the momentum of light [6,7]. In Barnett's theory, $\mathbf{g}_{M}$ is called as the canonical momentum density, but the single-photon value of the momentum is determined by the spatial shift of the field rather than by the single-photon expectation value of $\int \mathbf{g}_{\mathrm{M}} d^{3} r[6,7]$. In contrast to Barnett's theory, in our MP theory, all complications are avoided as the Minkowski momentum $p_{\mathrm{MP}}=n_{\mathrm{p}} \hbar \omega / c$ of the MP is also obtained for the single-photon expectation value calculated by using the total MP momentum density $\mathbf{g}_{\mathrm{MP}}=\rho_{\mathrm{a}} \mathbf{v}_{\mathrm{a}}+\mathbf{E} \times \mathbf{H} / c^{2}$.

In the literature, there exist also other attempts to explain the difference between the two different forms of the Minkowski momentum. According to Philbin [35] and the very recent works by Bliokh et al. $[47,48]$, the form $p_{\mathrm{M}}=n_{\mathrm{p}}^{2} \hbar \omega /\left(n_{\mathrm{g}} c\right)$ is obtained if one neglects certain dispersion-related terms in the momentum density while accounting for these terms gives the correct form $\tilde{p}_{\mathrm{M}}=n_{\mathrm{p}} \hbar \omega / c$, which equals the MP momentum of the present work in Eq. (23). These works did not however present any general splitting of the total Minkowski momentum to the field and the atomic MDW parts. In the studies of surface plasmon polaritons (SPPs) $[47,48]$, Bliokh et al. found that there is a current of electrons which accounts for the difference between the Abraham and Minkowski momenta. Therefore, one can expect that, in structures supporting SPPs, the MDW may correspond to the excess mass density due to the moving electrons. The concept of the covariant state of light and the related MDW are very general and expected to apply to the description of light propagation in any material structures.

\section{Expression of the optical force}

In the derivation of the covariant description of the light pulse, we have emphasized the coupled state of the field and matter as an internal property of the MP. Also, the OCD simulations are carried far from the interfaces so that the first term of the optical force density Eq. (9) including the gradient of the phase refractive index does not influence the momentum or the transferred mass of the light pulse. For this internal coupled state, we can forget various interface effects and consider only the second term of the optical force density in Eq. (9). The second term in Eq. (9) represents the generalization of the well-known Abraham force for a dispersive medium.

The full agreement between the MP and OCD models is obtained only if the Abraham force used in the OCD model is of the form given in Eq. (9). The interface term of the optical force then also obtains an unambiguous form because of the momentum conservation at the interfaces. Thus, the MP theory of light in a dispersive medium leads to a unique expression for the optical force.

\section{CONCLUSIONS}

We have generalized the recently developed MP theory of light for dispersive media assuming that the absorption and scattering losses are very small. The total momentum and the transferred mass of the light pulse were derived both using the MP quasiparticle model and the OCD model. In the OCD simulations, we have considered only solid dispersive media. However, the relation of the calculated momentum and the transferred mass to the phase and group refractive indices and to the pulse shape is very accurately the same for dispersive liquids. For liquids, the relaxation dynamics restoring the equilibrium in the medium is governed by the Navier-Stokes dynamics instead of the elasticity theory. The effect of the restoring force on the MP state of light is evidently very small and it is mainly related to the strain field losses. Detailed study of the MP theory of light in liquids is a topic of further work. The MP theory as formulated in this work and in Ref. [1] also applies for gases. There, in the OCD analysis, the relaxation dynamics is described by the Fokker-Planck equation. Note that the MP quasiparticle model appears to be universally valid independently on the phase of the medium. If we know the electromagnetic energy of the field and the velocity of light in the medium or, in the dispersive case, the 
dispersion relation of the medium, we can independently of the phase of the medium use the results in Table I to obtain the total momentum and the transferred mass of the light pulse.

In analogy to the case of a nondispersive medium, we found an unambiguous correspondence between the MP quasiparticle and OCD models. The present results generalize our ultimate solution of the Abraham-Minkowski controversy for a dispersive medium. The interesting feature in the MP theory of light in a dispersive medium is the atomic MDW and the related mass transfer which explains how the total momentum of light is shared between the field and medium atoms which move under the influence of the optical force. The mass transferred by the MDW makes our theory to fulfill Newton's first law and the covariance condition of the special theory of relativity. We have also proven that the covariance condition and the conservation laws jointly determine the expression of the optical force on the medium associated with a light pulse.

In analogy to our analysis of light propagation in a nondispersive medium, we also found in this work that the field and the MDW can be described using classical variables of the field and medium dynamics. This implies that the dynamical state of the medium is described entirely using phase phase, i.e., momentum and position related to each degree of freedom of the system. Therefore, since momentum and position are in classical physics unambiguously measurable, in principle to the desired degree of accuracy, we can experimentally determine at any moment how the momentum is shared between the field and the medium. This result is in contrast to many previous works on the momentum of light in a medium [5].

To facilitate the planning of measurements, we have also carried out simulations of how the displacement of atoms due to the MDW can be measured in a simple silicon waveguide structure. In these simulations, we have paid particular attention in the irradiance breakdown threshold of silicon, which is one of the main limiting factors in possible experimental setups as the electromagnetic energy density in the medium cannot be made arbitrarily large. The OCD model also allows for more detailed simulations accounting for the waveguide dispersion and the spreading of the pulse energy in the transverse direction. These simulations are left as a topic of further work.

\section{ACKNOWLEDGMENTS}

This work has in part been funded by the Academy of Finland under Contract No. 287074 and the Aalto Energy Efficiency Research Programme.
[1] M. Partanen, T. Häyrynen, J. Oksanen, and J. Tulkki, Photon mass drag and the momentum of light in a medium, Phys. Rev. A 95, 063850 (2017).

[2] L. D. Landau, E. M. Lifshitz, and L. P. Pitaevskii, Electrodynamics of Continuous Media (Pergamon, Oxford, 1984).

[3] U. Leonhardt, Momentum in an uncertain light, Nature (London) 444, 823 (2006).

[4] A. Cho, Century-long debate over momentum of light resolved?, Science 327, 1067 (2010).

[5] R. N. C. Pfeifer, T. A. Nieminen, N. R. Heckenberg, and H. Rubinsztein-Dunlop, Colloquium: Momentum of an electromagnetic wave in dielectric media, Rev. Mod. Phys. 79, 1197 (2007).

[6] S. M. Barnett, Resolution of the Abraham-Minkowski Dilemma, Phys. Rev. Lett. 104, 070401 (2010).

[7] S. M. Barnett and R. Loudon, The enigma of optical momentum in a medium, Philos. Trans. R. Soc. A 368, 927 (2010).

[8] U. Leonhardt, Abraham and Minkowski momenta in the optically induced motion of fluids, Phys. Rev. A 90, 033801 (2014).

[9] P. L. Saldanha and J. S. O. Filho, Hidden momentum and the abraham-minkowski debate, Phys. Rev. A 95, 043804 (2017).

[10] I. Brevik, Minkowski momentum resulting from a vacuummedium mapping procedure, and a brief review of Minkowski momentum experiments, Ann. Phys. 377, 10 (2017).

[11] T. Ramos, G. F. Rubilar, and Y. N. Obukhov, First principles approach to the Abraham-Minkowski controversy for the momentum of light in general linear non-dispersive media, J. Opt. 17, 025611 (2015).

[12] M. E. Crenshaw, Decomposition of the total momentum in a linear dielectric into field and matter components, Ann. Phys. 338, 97 (2013).
[13] M. Mansuripur, Resolution of the Abraham-Minkowski controversy, Opt. Commun. 283, 1997 (2010).

[14] B. A. Kemp, Resolution of the Abraham-Minkowski debate: Implications for the electromagnetic wave theory of light in matter, J. Appl. Phys. 109, 111101 (2011).

[15] M. Abraham, Zur Elektrodynamik bewegter Körper, Rend. Circ. Matem. Palermo 28, 1 (1909).

[16] M. Abraham, Sull'elettrodinamica di Minkowski, Rend. Circ. Matem. Palermo 30, 33 (1910).

[17] H. Minkowski, Die Grundgleichungen für die elektromagnetischen Vorgänge in bewegten Körpern, Nachr. Ges. Wiss. Goettingen, Math. Phys. K1., 53 (1908), reprinted in Math. Ann. 68, 472 (1910).

[18] G. K. Campbell, A. E. Leanhardt, J. Mun, M. Boyd, E. W. Streed, W. Ketterle, and D. E. Pritchard, Photon Recoil Momentum in Dispersive Media, Phys. Rev. Lett. 94, 170403 (2005).

[19] R. E. Sapiro, R. Zhang, and G. Raithel, Atom interferometry using Kapitza-Dirac scattering in a magnetic trap, Phys. Rev. A 79, 043630 (2009).

[20] R. V. Jones and J. C. S. Richards, The pressure of radiation in a refracting medium, Proc. R. Soc. London, Ser. A 221, 480 (1954).

[21] R. V. Jones and B. Leslie, The measurement of optical radiation pressure in dispersive media, Proc. R. Soc. London, Ser. A 360, 347 (1978).

[22] G. B. Walker and D. G. Lahoz, Experimental observation of Abraham force in a dielectric, Nature (London) 253, 339 (1975).

[23] W. She, J. Yu, and R. Feng, Observation of a Push Force on the end Face of a Nanometer Silica Filament Exerted by Outgoing Light, Phys. Rev. Lett. 101, 243601 (2008).

[24] L. Zhang, W. She, N. Peng, and U. Leonhardt, Experimental evidence for Abraham pressure of light, New J. Phys. 17, 053035 (2015). 
[25] N. G. C. Astrath, L. C. Malacarne, M. L. Baesso, G. V. B. Lukasievicz, and S. E. Bialkowski, Unravelling the effects of radiation forces in water, Nat. Commun. 5, 4363 (2014).

[26] A. Ashkin and J. M. Dziedzic, Radiation Pressure on a Free Liquid Surface, Phys. Rev. Lett. 30, 139 (1973).

[27] A. Casner and J.-P. Delville, Giant Deformations of a LiquidLiquid Interface Induced by the Optical Radiation Pressure, Phys. Rev. Lett. 87, 054503 (2001).

[28] I. Brevik, Experiments in phenomenological electrodynamics and the electromagnetic energy-momentum tensor, Phys. Rep. 52, 133 (1979).

[29] D. J. Griffiths, Introduction to Electrodynamics (Prentice-Hall, Upper Saddle River, NJ, 1998).

[30] P. W. Milonni and R. W. Boyd, Momentum of light in a dielectric medium, Adv. Opt. Photon. 2, 519 (2010).

[31] K.-E. Peiponen, E. M. Vartiainen, and T. Asakura, Dispersion, Complex Analysis and Optical Spectroscopy: Classical Theory (Springer, Berlin, 1999).

[32] A. Bedford and D. S. Drumheller, Introduction to Elastic Wave Propagation (Wiley, Chichester, 1994).

[33] G. Mavko, T. Mukerji, and J. Dvorkin, The Rock Physics Handbook (Cambridge University Press, Cambridge, England, 2003).

[34] C. Kittel, Introduction to Solid State Physics (Wiley, Hoboken, NJ, 2005).

[35] T. G. Philbin, Electromagnetic energy momentum in dispersive media, Phys. Rev. A 83, 013823 (2011).

[36] J. C. Garrison and R. Y. Chiao, Canonical and kinetic forms of the electromagnetic momentum in an ad hoc quantization scheme for a dispersive dielectric, Phys. Rev. A 70, 053826 (2004).

[37] J. Chen, Y. Wang, B. Jia, T. Geng, X. Li, L. Feng, W. Qian, B. Liang, X. Zhang, M. Gu, and S. Zhuang, Observation of the inverse Doppler effect in negative-index materials at optical frequencies, Nat. Photon. 5, 239 (2011).
[38] P. W. Milonni and R. W. Boyd, Recoil and photon momentum in a dielectric, Laser Phys. 15, 1432 (2005).

[39] A. Smith, B. Do, and M. Soderlund, Deterministic nanosecond laser-induced breakdown thresholds in pure and $\mathrm{Yb}^{3+}$ doped fused silica, Proc. SPIE 6453, 645317 (2007).

[40] See Supplemental Material at http://link.aps.org/supplemental/ 10.1103/PhysRevA.96.063834 for video files of the simulated MDW and atomic displacements in linearly and nonlinearly dispersive media.

[41] H. H. Li, Refractive index of silicon and germanium and its wavelength and temperature derivatives, J. Phys. Chem. Ref. Data 9, 561 (1980).

[42] CRC Handbook of Chemistry and Physics, edited by D. R. Lide (CRC, Boca Raton, FL, 2004).

[43] M. A. Hopcroft, W. D. Nix, and T. W. Kenny, What is the Young's modulus of silicon?, J. Microelectromech. Syst. 19, 229 (2010).

[44] B. Cowan, Optical damage threshold of silicon for ultrafast infrared pulses, in Advanced Accelerator Concepts: 12th Advanced Accelerator Concepts Workshop, AIP Conf. Proc. No. 877, edited by M. Conde and C. Eyberger (AIP, Melville, NY, 2006).

[45] C. Schinke, P. C. Peest, J. Schmidt, R. Brendel, K. Bothe, M. R. Vogt, I. Kröger, S. Winter, A. Schirmacher, S. Lim, H. T. Nguyen, and D. MacDonald, Uncertainty analysis for the coefficient of band-to-band absorption of crystalline silicon, AIP Adv. 5, 067168 (2015).

[46] M. A. Green, Self-consistent optical parameters of intrinsic silicon at $300 \mathrm{~K}$ including temperature coefficients, Sol. Energ. Mat. Sol. Cells 92, 1305 (2008).

[47] K. Y. Bliokh, A. Y. Bekshaev, and F. Nori, Optical Momentum, Spin, and Angular Momentum in Dispersive Media, Phys. Rev. Lett. 119, 073901 (2017).

[48] K. Y. Bliokh, A. Y. Bekshaev, and F. Nori, Optical momentum and angular momentum in complex media: From the AbrahamMinkowski debate to unusual properties of surface plasmonpolaritons, New J. Phys. 19, 123014 (2017). 